$2=11-50 \% ; 3=51-100 \%)$ and intensity of staining $(0-3+)$. A $5 \mathrm{hmC}$ score was calculated by multiplying the percentage and intensity scores (range $0-9$ ), and a score of $<3$ was considered deficient for $5 \mathrm{hmC}$.

Results: Normal and cirrhotic liver parenchyma showed predominantly intact expression of $5 \mathrm{hmC}$, with $5 \mathrm{hmC}$ scores ranging from 3-9. In some cases, staining was weak (1+) but present in the majority of cells. In contrast, expression of $5 \mathrm{hmC}$ was deficient in $93 \%(25 / 27)$ of HCC cases. The intensity and percentage of $5 \mathrm{hmC}$ expression in HCC were dramatically reduced compared to surrounding benign hepatocytes or cirrhotic nodules. No significant difference in $5 \mathrm{hmC}$ expression was seen between $\mathrm{HCC}$ tumor grades (Chi-Square test, $\mathrm{p}=0.19) .83 \%(5 / 6)$ cases of HA exhibited intact $5 \mathrm{hmC}$ expression, with most cases showing slightly reduced $5 \mathrm{hmC}$ expression compared to neighboring benign hepatocytes.

Conclusions: Nearly all cases of HCC in our cohort were deficient for $5 \mathrm{hmC}$ expression, compared to intact $5 \mathrm{hmC}$ expression in normal/cirrhotic surrounding hepatocytes and HA cases. $5 \mathrm{hmC}$ could serve as an important diagnostic tool in the diagnosis of HCC, and suggests an underlying role for epigenetic dysfunction in liver tumorigenesis.

\section{Neuropathology and Ophthalmic Pathology}

\section{$1715 \quad$ BAP1 Mutations in Rhabdoid Meningiomas}

Malak Abedalthagafi. King Fahad Medical City, Riyadh, Saudi Arabia; King Abdulaziz for Science and Technology, Riyadh, Saudi Arabia; Harvard Medical School-Brigham and Women's Hospital, Boston, MA.

Background: Patients with meningiomas have widely divergent clinical courses. Some entirely recover following surgery alone while others have relentless tumor recurrences which can lead to death. This clinical conundrum is exemplified by rhabdoid meningiomas which are designated in the World Health Organization (WHO) Classification of Tumors as the highest grade of meningioma, despite only a subset actually following a highly aggressive clinical course. Patient management decisions are further exacerbated by high rates of interobserver variability amongst diagnosticians, biased against missing possibly aggressive tumors. Objective molecular determinants are therefore needed to guide clinical decision-making and to prevent both over-and under- treatment.

Design: We performed sequencing of cancer related genes in 26 meningioma with rhabdoid features and evaluated BAP1 protein expression by immunohistochemistry in 336 meningiomas. We assessed outcomes, germline status and family history in patients with BAP1-negative rhabdoid meningiomas.

Results: The tumor suppressor gene BAP1 (BRCA1-associated protein 1) is inactivated in high-grade, rhabdoid meningiomas. Patients with BAP1-negative rhabdoid meningiomas had reduced time to recurrence compared with patients with BAP1retained rhabdoid meningiomas (Kaplan Meier analysis, 26 months vs 116 months, $\mathrm{p}<0.001$ ). A subset of patients harbored germline BAP1 mutations, indicating that rhabdoid meningiomas can be a harbinger of the BAP1 cancer predisposition syndrome. Conclusions: We define a subset of aggressive rhabdoid meningiomas that can be recognized using routine laboratory tests. We implicate ubiquitin deregulation in the pathogenesis of these high-grade malignancies. In addition, we show that familial and sporadic BAP1-mutated rhabdoid meningiomas are clinically aggressive, requiring intensive clinical management.

1716 Diverse Patterns of Involvement in Intraocular Lymphoma: Learning from Four Enucleation Specimens

Osamah AL Badri, Diva Salomao, Joaquin Garcia, Jose Pulido. Mayo Clinic, Rochester, MN.

Background: Intraocular lymphomas are rare and often pose challenges both clinical and diagnostically. The majority of cases are diagnosed on vitrectomy specimens; enucleation is seldom performed for diagnostic purposes. Most enucleated specimens are submitted as post-treatment blind painful eye. Herein,we describe the diverse patterns of involvement in enucleation specimens involved by intraocular lymphomas, 2 primary vitreoretinal lymphomas naïve of treatment and 2 systemic lymphomas with intraocular involvement.

Design: Computer search of Anatomic Pathology files (January 1994 to November 2015) revealed 4 enucleation specimens with intraocular lymphoma. Electronic medical records were reviewed for demographics, clinical findings, laboratory and radiographic studies results, and indication for enucleation. The slides in all cases were reviewed. Results: Two cases were large B-cell vitreoretinal lymphomas, naïve of treatment, in 80 and 75 year old women treated for uveitis with no success. Both specimens showed extensive retinal and sub-retinal infiltrates of large atypical B-lymphocytes (positive for CD20, Bc16, MUM1 and MYC, among several markers tested). Rare neoplastic cells were present in the vitreous, and the choroid had only reactive infiltrate of small lymphocytes (CD3 positive T-cells). One case showed MYD88L265P mutation by PCR. Remaining two cases were on patients with systemic lymphoproliferative disorders. The first, a 65 year old man with underlying CLL, complaining of floaters, was diagnosed with large B-cell lymphoma on vitrectomy, consistent with intra ocular Richter's transformation. The enucleated eye showed treatment effect and residual lymphoma involving retina and choroid. The last case, a 69 year old woman with stage III diffuse large B-cell lymphoma in remission, developed blurred vision on left eye, was treated for panuveitis without improvement, and later diagnosed on choroidal biopsy with ocular involvement by large B-cell lymphoma. The enucleated specimen showed focal residual lymphoma located in the choroid.

Conclusions: Different patterns of ocular tissue involvement were observed in these four cases when we compared primary and secondary intraocular lymphomas. The paucity of vitreous tumor cells in the 2 cases of vitreoretinal lymphoma supports the diagnostic challenge one faces in some vitrectomy specimens. In addition, one of our cases exemplifies the unusual occurrence of Richter's transformation in the eye.

\section{Differential Exon Expression Analysis in Glioblastomas} Multiforme by Quantitative Statistics

Jaudah A Al-Maghrabi, Hans-Juergen Schulten, Deema Hussein, Sajjad Karim, Fahad Al-Ghamdi, Saleh Baeesa, Mohammed Bangash, Mohammad Al-Qahtani. Faculty of Medicine, King Abdulaziz University, Jeddah, Saudi Arabia; Center of Excellence in Genomic Medicine Research, Jeddah, Saudi Arabia; King Abdulaziz University, Jeddah, Saudi Arabia.

Background: Glioblastoma multiforme (GBM) is afflicted with dismal prognosis despite the fact that major aspects of its molecular genetics have been comprehensively investigated. Although differential exon usage is known to mediate distinct biological functions, until now only a few expression studies have investigated exon splicing events in GBM leaving a gap in the knowledge of the molecular genetics of this disease. Design: We conducted a quantitative statistical analysis on microarray expression datasets which are curated by the Gene Expression Omnibus (GEO) and which used HuGene 1.0 ST microarrays for analysis. This type of whole transcript microarray interrogates with a set of 764,885 probes 36.079 annotated reference sequences representing 253002 exons (NCBI build 36). The data sets consisted of 71 GBM that were compared to 14 non-GBM gliomas comprising astrocytoma, oligoastrocytoma, and oligodendroglioma cases. A p-value with a false discovery rate (FDR) $<0.05$ and an exon-based fold change $>2$ ( $>10$ for a comprehensive short list) were used as a threshold of significance for differential expression (Transcriptome Analysis Console v3.1, Affymetrix, Santa Clara, CA).

Results: We identified in our quantitative analysis a number of genes which contained one or more exons that were both, differentially expressed between GBM and nonGBM gliomas and in the GBM group comparably lower expressed than other exons from the same gene. Among the identified genes were semaphorin 3D (SEMA3D), mohawk homeobox $(M K X)$, RALBP1 associated Eps domain containing 2 (REPS2), and cholecystokinin $(C C K)$. Different splice variants for these genes are described. In addition, a number of identified genes are likely to harbor exons that are differentially expressed in non-GBM gliomas or differentially expressed in both, GBM and nonGBM gliomas.

Conclusions: Combining publicly available expression datasets and using for statistical comparison non-GBM gliomas which are histologically related to GBM, we were able to identify a number of candidate genes that contain differential expressed exons. Of notice, SEMA3D which is a secreted semaphorin receptor binding protein is known to mediate breakdown and paralysis of neuronal growth cones and to inhibit glioblastoma cell proliferation in vivo. In further studies we have to sustain our in silico findings in clinical samples and functional assays.

\section{Focal Cortical Dysplasia-Associated Low-Grade Glioma:} Expending the BRAF Mutations Concept

Azniv Azar, Siraj M El Jamal, Youssef Al Hmada, Ali G Saad. University of Mississippi Medical Center, Jackson, MS.

Background: It has been shown that focal cortical dysplasia (FCD) and gangliogliomas originate from a common precursor that undergoes abnormal glioneuronal development. Furthermore, it has been recently demonstrated the presence of $B R A F$ mutations in low-grade epilepsy-associated tumors. Here we demonstrate that $B R A F$ mutations are present, not only in the tumor, but also in the adjacent dysplastic neurons.

Design: Electronic records were searched for pediatric patients with FCD and associated low-grade glioma in the last 10 years. In all patients, slides were reviewed and representative sections were selected. BRAF molecular status was evaluated in the low-grade glioma, in the FCD, and in the adjacent normal cerebral cortex. For this purpose, DNA was extracted from paraffin-embedded tissue blocks using laser capture microdissection. In addition to the associated tumor, normal and dysplastic neurons were microdissected from normal and dysplastic cerebral cortex, respectively.

Results: The search resulted in 7 cases of concomitant FCD and low-grade gliomas. The gliomas were as follows: 2 diffuse astrocytoma (DA) WHO grade II, 3 ganglioglioma (GG) WHO Grade I, and 2 pleomorphic xanthoastrocytoma (PXA) WHO grade II. The patients consisted of 5 males and 2 females (Age range: 5-13.5 years; average: 8.6 years). Six tumors were in the temporal lobe and one (PXA) in the frontal lobe. BRAF mutation (V600E) was present in two DA and one PXA. In both cases, $B R A F$ mutations were concomitantly present in the associated FCD. In one DA and one GG, BRAF mutation was present only in the tumor while the adjacent dysplastic neurons and normal neurons showed no mutation. No BRAF mutation was noted in the normal cerebral cortex in all 7 patients. FCD consisted of 3 cases of type IIa and 4 cases of type IIIb.

Conclusions: Our results suggest that FCD and FCD-associated glioma represent a spectrum in the "focal cortical development malformation" concept. Larger studies are required in order to understand the significance of $B R A F$ mutations in FCD as well as to investigate the role of $B R A F$ mutations in epilepsy-associated tumors and FCD and their role as prognostic tumor behavior marker.

1719 Pineocytomas

Tiffany Baker, Iya Znoyko, Daynna J Wolff, Cynthia Welsh. Medical University of South Carolina, Charleston, SC.

Background: Pineocytomas are well-differentiated tumors of the central nervous system that arise from the pineal gland parenchyma. Their rarity, bland histologic features, and potential to demonstrate cystic architecture, which may render them indistinguishable from non-neoplastic pineal cysts via imaging, all contribute to the persistent diagnostic 
dilemma associated with these tumors. Molecular techniques continue to change the diagnostic landscape of medicine. Studies investigating the genetics of pineocytomas have included conventional cytogenetics, comparative genome hybridization, gene expression microarray, and investigations into $\mathrm{p} 53$ mutations. The sample sizes in these studies reached an upper limit of five pineocytoma specimens. To our knowledge, no study demonstrates the application of single nucleotide polymorphism (SNP) microarray to pineocytomas. The purpose of the study was to perform SNP microarray on formalinfixed paraffin-embedded (FFPE) pineal lesions that were diagnosed as pineocytoma based on histopathology in order to identify cytogenetic abnormalities that could aid in the diagnosis of these lesions.

Design: Fourteen recent cases of pineocytoma were identified at our institution, and 12 of these were selected for microarray analysis. Archival FFPE tissue from these cases was acquired for SNP microarray analysis. Hematoxylin and eosin stained slides corresponding to the FFPE tissue blocks were examined by a board certified neuropathologist for the purpose of selecting tissue regions for analysis. Genomic DNA was extracted from the tissue, and microarray-based chromosome analysis was performed. Copy number and genotype data were analyzed.

Results: Analyzed cases came from female patients 19 to 54 years old. Their reported symptoms included headache, dizziness, tinnitus, various visual disturbances, speech disturbances, numbness and/or tingling of extremities, nausea, and memory loss. Pineocytomas ranged in size from 1.1 to $1.8 \mathrm{~cm}$ in greatest linear extent. SNP microarray analysis revealed no copy number alterations.

Conclusions: To our knowledge, the present study represents the largest molecular study of pineocytomas to date and the first to employ SNP microarray analysis for the purpose of further characterizing these potentially ambiguous lesions. Our data indicate that these tumors exhibit no copy number changes and are thus genetically identical to normal pineal gland at this resolution. This information may be useful to clinicians when counseling patients with suspected pineocytomas.

\section{The Histologic and Molecular Features of Pediatric and Adult} Gliomas with Alterations in FGFR1 and FGFR3

Tejus A Bale, Sanda Alexandrescu, Monica Manam, Neal I Lindeman, Adrian Dubuc, Azra H Ligon, Keith L Ligon. Brigham and Women's Hospital, Boston, MA; Boston Children's Hospital, Boston, MA.

Background: FGFR alterations have been previously described in both low and highgrade primary brain tumors and include FGFR1 missense mutations and intragenic duplication, fusions (FGFR1-TACC1, FGFR3-TACC3) and FGFR2 copy number alterations. Though observed in only $5-10 \%$ of gliomas, these alterations are of great interest because of their targetable therapeutic potential. It is not clear if a distinct tumor subtype exists among gliomas with FGFR alterations. We performed a systematic histologic, cytogenetic, and molecular characterization of gliomas with FGFR1 and FGFR3 alterations.

Design: Adult and pediatric gliomas with alterations in FGFR1 and FGFR3 on clinical high-resolution array comparative genomic hybridization (OncoCopy, OC) were retrospectively identified. Histology was reviewed by two neuropathologists. Cytogenetic and molecular information were reviewed by a panel of cytogeneticists, molecular pathologists and neuropathologists. Targeted exome sequencing (OncoPanel, 275 genes) with BreaKmer analysis were compiled and analyzed for recurrent events. Results: A total of 36 cases were identified, 31 adults and 5 pediatric. Four categories of molecular alterations emerged: focal FGFR1 gains $(n=4)$, focal FGFR3 gains $(n=12)$, FGFR3 rearrangements (FGFR3-TACC3, FGFR3-FAM104a, FGFR3-STON2, n=17), and FGFR1-TACC1 rearrangement $(\mathrm{n}=3)$. All tumors with FGFR1 and FGFR3 gains, $33 \%$ of tumors with FGFR1-TACC1, and $88 \%$ of FGFR3 rearranged cases had highgrade histology, including $80 \%$ of pediatric cases. 2 cases with FGFR3-TACC3 and 2 cases with FGFR1-TACC1 displayed low-grade glioma histology. All tumors with FGFR1 gains and 33\% of tumors with FGFR3 gains demonstrated pleomorphic, bizarre cytomorphology and multinucleation. Tumors with FGFR3 and FGFR1 rearrangements displayed exuberant endothelial proliferation ( $64.7 \%$ of cases) and microcalcifications $(47 \%)$. Co-occurring copy number alterations included polysomy 7 , monosomy 10,13 , 22, 9p losses with 1-2 copy loss of CDKN2A. Recurrently altered genes included RB1, PTEN, TP53, and CDKN2A. FGFR3 gains co-occurred with ATRX frameshift mutations and IDH1 mutations in $25 \%$ of cases.

Conclusions: FGFR1 and FGFR3 are altered in pediatric and adult gliomas in mutually exclusive events (focal gains, fusions), and in our experience occur most commonly in tumors displaying histologic and cytogenetic signatures of high grade tumors. Moreover, many tumors harboring these alterations display distinct histologic and molecular features with possible biological and clinical significance.

1721 Retrospective Analysis of Molecular and Immunohistochemical Characterization of $\mathbf{3 8 1}$ Primary Brain Tumors

Leomar Ballester, Gregory N Fuller, Suzanne Z Powell, Erik P Sulman, Keyur P Patel, Rajyalakshmi Luthra, Mark J Routbort. Houston Methodist Hospital, Houston, TX; The UT MD Anderson Cancer Center, Houston, TX.

Background: The classification of brain tumors has traditionally depended on microscopic examination of hematoxylin and eosin (H\&E) stained tissue sections The increased understanding of clinically-relevant genetic alterations has led to the incorporation of molecular signatures as part of the diagnosis of brain malignancies. Advances in sequencing technologies over the past years have facilitated the routine use of next generation sequencing (NGS) assays in many clinical laboratories.

Design: We performed a retrospective analysis of sequencing results for 381 brain tumors that were tested by NGS at our institution using a validated, commercially available panel. The results of the NGS assay were analyzed in conjunction with the results of immunohistochemical stains.
Results: A genetic alteration was detected in $\sim 2 / 3$ of the cases. The most commonly mutated genes in our study were TP53 (37.2\%), IDHI (29.4\%), PIK3CA (8\%), PTEN $(8 \%)$ and $E G F R(7.5 \%)$. BRAF mutations were detected in $~ 3 \%$ of the cases, including $50 \%$ of gangliogliomas and $\sim 20 \%$ of gliosarcomas. No mutations were detected in 6 medulloblastomas. A PIK3CA and a CTNNB1 mutation was detected in a rosette forming glioneuronal tumor and one adamantinomatous craniopharyngioma, respectively. Approximately $23 \%$ of cases showed evidence for amplification of at least one of the genes included in the NGS panel.

Conclusions: This analysis demonstrates the utility of NGS analysis for detecting genetic alterations in brain tumors in the clinical setting.

1722 The Importance of Peri-Adventitial Soft Tissue Examination in Temporal Artery Biopsies for Suspected Giant Cell Arteritis

Paula Blanco, James Farmer. University of Ottawa, Ottawa, ON, Canada.

Background: Temporal artery biopsy (TAB) is the gold standard diagnostic modality for giant cell arteritis (GCA). Transmural inflammation in the temporal artery, usually granulomatous and centered on the internal elastic lamina, is the hallmark for the diagnosis. Therefore, it is not an uncommon practice in many centers to "skeletonize" the main artery and discard the soft tissue surrounding it at the time of performing the biopsy. Careful examination of the peri-adventitial tissue, however, may reveal critical additional pathological findings that otherwise would have been missed.

Design: We report 5 cases where examination of peri-adventitial soft tissue in TAB performed to rule out GCA allowed for additional pathologic diagnoses. None of the cases had a pre-biopsy clinical suspicion of the final diagnosis.

Results: Patient 1 is a 66-year-old man with amaurosis fugax, elevated ESR and C-reactive protein (CRP), and systemic symptoms. TAB revealed small vessel vasculitis with predominance of eosinophils. Eosinophilic granulomatosis with polyangiitis was diagnosed after systemic work up.

Patient 2 is a 83 -year-old woman with polymyalgia rheumatica and chronically elevated ESR and CRP. She presented with headaches and jaw claudication. TAB showed small vessel vasculitis with predominance of neutrophils. She was later diagnosed with p-ANCA associated vasculitis.

Patient 3 is a 68 -year-old woman with intermittent right temple pain and no other symptoms. ESR was mildly elevated. TAB showed small vessel vasculitis with predominance of neutrophils. She was diagnosed with p-ANCA associated vasculitis. Patient 4 is a 82 -year-old man with headache, blurry vision, scalp tenderness and elevated ESR and CRP. Bilateral TAB were negative for inflammation in the main artery but a small branch on the left side showed unequivocal transmural lymphocytic inflammation centered around the internal elastic lamina consistent with active arteritis. Patient 5 is a 76-year-old man with scalp tenderness, bitemporal headaches and an elevated ESR. TAB showed healed arterial injury in the main artery and an atypical population of lymphocytes in the peri-adventitial tissue. Immunohistochemistry showed findings suggestive of a lymphoproliferative disorder. He was later diagnosed with chronic lymphocytic leukemia.

Conclusions: We conclude that surgeons should always include peri-adventitial soft tissue in all TAB for suspected GCA, and pathologists should always carefully examine it regardless of the findings in the main artery.

\section{Morphologic Features of Inflammatory Myopathy Associated with Anti-Aminoacyl-tRNA Synthetase Antibodies}

Bruna Cerbelli, Annalinda Pisano, Serena Colafrancesco, Marco Biffoni, Silvia Berni, Valeria Ricceri, Roberta Priori, Guido Valesini, Giulia d'Amati, Carla Giordano. Sapienza University, Rome, Italy.

Background: Antisynthetase syndrome (ASS) is characterized by antibodies agains aminoacyl-tRNA synthetases (ARS), interstitial lung disease, arthritis, cutaneous manifestations and a peculiar form of myositis restricted to the peripheral portion of the muscle fascicle. This pattern of topographic distribution of muscle damage in ASS may be misinterpreted as dermatomyositis (DM) on muscle biopsy.

Design: To provide clues for the differential diagnosis, we compared the morphological, immunohistochemical and histoenzymatic features of muscle biopsies from patients with ASS $(n=11)$ and DM $(n=5)$. In addition, we evaluated both capillary density and diameter in DM, ASS, and control samples $(n=5)$.

Results: The main histologic finding in muscle from ASS patients was the necrosis of muscle fibers restricted to the peripheral portion of the fascicle $(72 \%$ of cases $)$, concomitant with inflammatory infiltrate (CD68+, CD8+). Necrosis was either extensive $(27 \%)$ or focal $(45,4 \%)$, and was always associated with regeneration, as shown by the widespread expression of neonatal myosin in the perifascicular region. Differently from ASS, in DM perifascicular atrophy (i.e. small non-necrotic fibers not expressing neonatal myosin) was the most peculiar aspect ( $100 \%$ of cases), while necrosis and regeneration were limited to scattered fibers. Interestingly, atrophic fibers in DM did not stain with cytochrome c oxydase (COX), pointing to mitochondrial dysfunction. Scattered COXdeficient fibers were observed also in ASS, both in the central and peripheral portion of the fascicle. This feature was prominent in two patients, and was associated with mitochondrial DNA deletions. Perimysial and perivascular inflammation, sarcolemmal complement deposition, and sarcoplasmic stain for major histocompatibility complex class I (MHCI), were common both in ASS and DM. Reduced density (up to 50\%) and dilation (up to $30 \%$ ) of capillaries were observed both in ASS and DM as compared to controls. These features were more evident in the perifascicular region and in ASS correlated with the severity of necrosis.

Conclusions: Perifascicular necrosis and abundant regenerating fibers characterize ASS-associated myositis. Scattered COX-deficient fibers may be a prominent feature. Perifascicular, COX-deficient atrophic fibers with scarce necrosis and regeneration are typical of DM. 
1724 Upregulated MiR-125, MiR-181d and MiR-221 Expression Levels Are Associated with Poor Prognosis in Glioblastoma Patients with Unmethylated MGMT Promoter

Yen-Ying Chen, Chih-Yi Hsu, Donald M-T Ho. Taipei Veterans General Hospital, Taipei, Taiwan.

Background: Glioblastoma (GBM) is the most malignant primary brain tumor with a poor prognosis. Several microRNAs have recently been identified as potential prognostic biomarkers in GBMs. However, the previous studies are performed on relatively small sample sizes, and some important prognostic factors such as IDH-1 status, TMZ treatment and O-6-methylguanine-DNA methyltransferase (MGMT) promoter methylation status are not completely included.

Design: One hundred and fourteen patients with primary GBM who had received TMZ chemotherapy with concomitant radiotherapy and had adequate follow-up data were included in the study. We analyzed the expression levels of several prognosis-related miRNAs (miR-125, miR-181d, miR-221, miR-222 and miR-224) and their relationships with survival in GBM patients. The expression levels were determined by real-time quantitative RT-PCR. MGMT promoter methylation status was also examined by methylation-specific PCR (MSP).

Results: Up-regulated expression of miR-125, miR-181d, miR-221 and miR-224 were noted in $13.2 \%, 5.3 \%, 12.3 \%, 32.5 \%$ and $78.9 \%$ GBMs. The expression levels of miRNAs were not significantly different by MGMT promoter methylation status. MiR-125, miR-181d and miR-221 had prognostic impact on MGMT unmethylated (MSP-) GBMs. Upregulation of any of these three miRNAs was significantly associated with shorter survival in MGMT MSP- GBMs (figure 1).

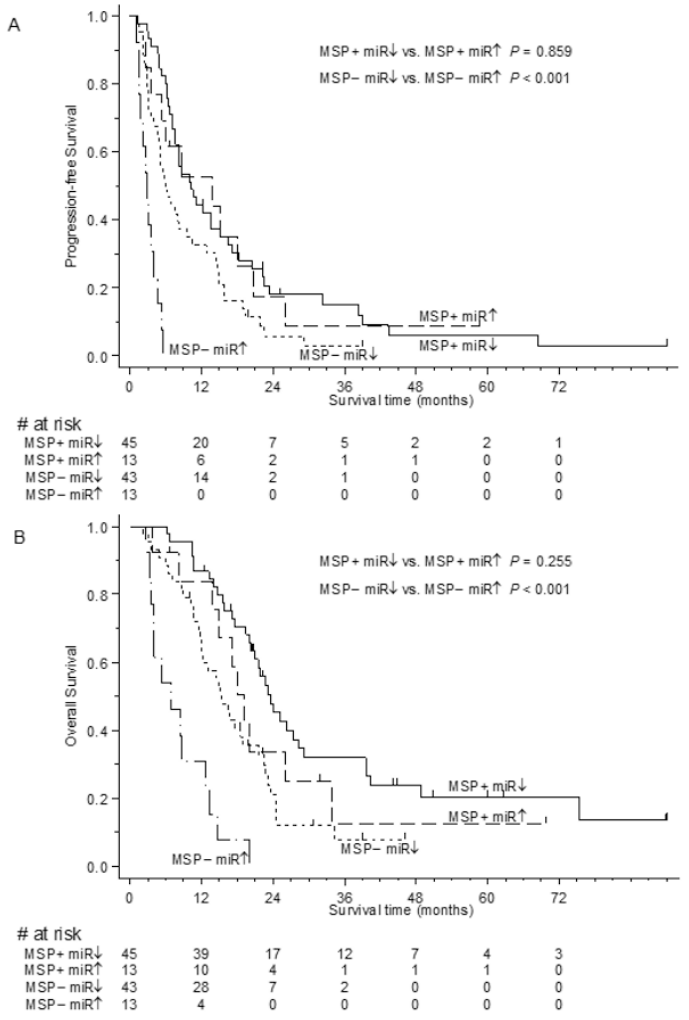

Their impact on survival remains statistically significant in multivariate anlyses with adjustment of age, gender, KPS, extent of resection, bevacizumab treatment, IDH1 status and MGMT methylation status (table 1). Table 1. Multivariate analyses of median survival by the expression of miRNAs (data presented as hazard ratio (P))

\begin{tabular}{|l|l|l|}
\hline MiRNA & Progression Free Survival & Overall Survival \\
\hline miR-125 & $1.902(0.036)$ & $2.221(0.012)$ \\
\hline miR-181d & $2.417(0.045)$ & $3.032(0.014)$ \\
\hline miR-221 & $1.797(0.066)$ & $2.039(0.032)$ \\
\hline miR-222 & $0.875(0.540)$ & $0.899(0.661)$ \\
\hline miR-224 & $0.890(0.669)$ & $0.838(0.546)$ \\
\hline
\end{tabular}

Conclusions: MiR-125, miR-181d and miR-221 are useful in predicting poor prognosis in MGMT unmethylated GBMs.

Distinctive CA9 Expression Patterns in Clear Cell, Microcystic and Angiomatous Meningioma Variants

Rati Chkheidze, Charles L White, Kimmo Hatanpaa, Chunyu Cai. UT Southwestern Medical Center, Dallas, TX.

Background: Carboxic anhydrase IX (CA9) is a hypoxia-induced protein overexpressed in many tumor types and associated with worse disease outcome. Expression of CA9 in meningiomas has been described as zonal, mutually exclusive to blood vessels, and associated with high grade histology. Clear cell, angiomatous, and microcyctic meningiomas are three uncommon variants that are all rich in blood vessels but with diverse clinical outcomes. Observation from isolated cases demonstrated unique CA9 expression patterns in these variants. In this study, we systematically compare the CA9 expression patterns of these three variants to conventional meningiomas.

Design: We Searched UTSW neuropathology database from 2000 to 2015 and identified meningiomas with prominent angiomatous $(\mathrm{N}=10)$, microcystic $(\mathrm{n}=7)$, or pure clear cell $(\mathrm{n}=6)$ morphologies. Immunostain for CA9 was performed on complete sections on all cases. The results were compared to those of 35 conventional atypical meningiomas. Results: In conventional meningiomas (meningotheloid, fibroblastic, transitional), CA9 staining is zonal, hypoxia restricted, and mutually exclusive to blood vessels. Strong expressions are typically seen in perinecrotic cells and areas with small cell change. All clear cell meningiomas showed complete or near complete absence of CA9 expression. Microcystic meningiomas, on the contrary, demonstrated uniform, diffuse CA9 positivity. Angiomatous meningiomas were more variable, most cases contained intermixed conventional or microcystic meningioma areas. Nine were negative for CA9 in the confluent vascular areas, one case showed diffuse positive staining similar to the micorcystic variant.

Conclusions: Our data show that CA9 staining demonstrates an all versus none expression pattern in microcystic and clear cell meningiomas, respectively, as opposed to a zonal, hypoxia restricted pattern in conventional meningiomas. The diffuse staining patterns irrespective of hypoxia gradient suggest a constitutive activation or inactivation of hypoxia pathway proteins in these meningioma variants. The distinctive staining patterns may aid in the diagnosis of equivocal cases. These data also point out potential pitfalls in using CA9 as a general poor prognosis marker in meningiomas.

1726 CRX and FOXJ1 Immunohistochemistry Differentiates Papillary Tumors of the Pineal Region from Pineal Parenchymal Lesions

Shannon Coy, Sonika Dahiya, Alexandre Vasiljevic, Sandro Santagata. Brigham and Women's Hospital, Boston, MA; Washington University School of Medicine, St. Louis, MO; Wertheimer Hospital, Lyon, France.

Background: Papillary tumors of the pineal region (PTPR) are rare neuro-epithelial tumors found in the pineal region. Despite their anatomic association with the pineal region and frequent histologic similarity to lesions of the pineal gland parenchyma; PTPR have transcriptional and ultrastructural features consistent with an origin in the sub-commissural organ. CRX immunohistochemistry (IHC) has been shown to specifically label benign pineal gland and pineal parenchymal lesions, but does not label ependymal cells. FOXJ1 is a master regulator of motile ciliogenesis which is highly expressed in cells of ependymal origin, but is not known to be expressed in pineal gland. We hypothesized that PTPR would show differential expression of CRX and FOXJ1 compared to pineal parenchymal lesions.

Design: We reviewed a diverse series of pineal region tumors including pineal parenchymal lesions and PTPR, as well as ependymomas, meningiomas, pilocytic astrocytomas, and medulloblastomas. All cases were stained using IHC for CRX. A subset of cases was stained with FOXJ1.

Results: CRX IHC strongly and diffusely labels the nuclei of benign pineal gland $(9 / 9,100 \%)$ and all lesions of pineal parenchymal origin, including pineal cysts $(4 / 4$, $100 \%)$, pineocytomas $(9 / 10,90 \%)$, pleomorphic pineocytomas $(2 / 2,100 \%)$, PPTIDs $(14 / 14,100 \%)$, and pineoblastomas $(5 / 5,100 \%)$. FOXJ1 IHC was negative $(0 \%$ of cells) in pineal gland and lesions of pineal parenchymal origin. CRX is negative in $75 \%$ of PTPR cases $(9 / 12)$, with only rare expression ( $<5 \%$ of cells) in the remaining cases. FOXJ1 is diffusely and strongly positive in $100 \%$ of cases of PTPR examined. CRX is negative in all cases of ependymoma $(0 / 44,0 \%)$, meningioma $(0 / 143,0 \%)$, and pilocytic astrocytoma $(0 / 23,0 \%)$. A subset of medulloblastoma cases $(22 / 49,45 \%)$ showed at least focal staining with CRX.

Conclusions: IHC for CRX and FOXJ1 robustly differentiates PTPR from benign pineal gland and lesions of pineal parenchymal origin. Our findings support the hypothesis that PTPR arise in the sub-commissural organ rather than the pineal gland. Given the marked histologic overlap of lesions in this anatomic region, IHC for CRX and FOXJ1 will likely be broadly applicable for evaluation of lesions of the pineal region.

1727 Loss of H3K27me3 Is a Poor Prognostic Factor for Meningioma Areli K Cuevas-Ocampo, David Raleigh, Ashley Wu, Bryan Tomlin, Joshua Menke, Gerald Reis, Arie Perry, Tarik Tihan, Melike Pekmezci. University of California in San Francisco, San Francisco, CA; Memorial Regional Hospital, Hollywood, FL.

Background: Although genetic alterations in meningiomas are relatively wellcharacterized, our understanding of meningioma epigenetics is incomplete. Trimethylation of lysine 27 at histone 3 (H3K27me3) is associated with either poor or favorable prognosis in various tumors. The aim of this study is to determine whether $\mathrm{H} 3 \mathrm{~K} 27 \mathrm{me} 3$ loss has prognostic implications in meningiomas, which is currently unknown.

Design: We retrospectively identified patients treated for meningioma from 1997 to 2014 with available tissue and clinical information. Immunohistochemical stains for $\mathrm{H} 3 \mathrm{~K} 27 \mathrm{me} 3$ were scored as loss (staining in $<50 \%$ cells), partial loss $(50-95 \%)$ or retained $(>95 \%)$. We analyzed the association of $\mathrm{H} 3 \mathrm{~K} 27 \mathrm{me} 3$ status with local recurrence-free survival (LRFS) and overall survival (OS) by the Kaplan-Meier method and log-rank tests. Relationships between LRFS or OS and age, sex, grade, setting (primary or recurrent), multiplicity, extent of resection and $\mathrm{H} 3 \mathrm{~K} 27 \mathrm{me} 3$ status were analyzed by multivariate regression. $\mathrm{P} \leq 0.05$ was considered statistically significant. Results: 230 meningiomas from 218 patients with a median age of 60 years (range 19-87) were included. Median follow up was 4.2 (1-15) years. H3K27me3 loss was associated with grade III and recurrent meningiomas (Table 1), but not with age, sex, location, or MIB1 index. On univariate analyses, H3K27me3 loss was associated with reduced LRFS and OS, and partial loss was associated with reduced OS. On 
multivariate analysis, grade III histology and subtotal resection, but not H3K27me3 loss, were associated with reduced LRFS. H3K 27 me 3 loss, grade III histology, recurrent meningiomas and age were associated with impaired OS on multivariate analysis.

\begin{tabular}{|c|c|c|c|}
\hline \multicolumn{4}{|l|}{ Table 1. H3K27me3 expression in meningiomas } \\
\hline & Retained (n=106) & Partial loss $(\mathrm{n}=86)$ & Loss $(\mathrm{n}=38)$ \\
\hline Grade & & & \\
\hline I (n=128) & $62(48 \%)$ & $51(40 \%)$ & $15(12 \%)$ \\
\hline II $(\mathrm{n}=79)$ & $36(46 \%)$ & $31(39 \%)$ & $12(15 \%)$ \\
\hline III $(\mathrm{n}=23)$ & $8(34 \%)$ & $4(17 \%)$ & $11(49 \%)$ \\
\hline Setting & & & \\
\hline Primary $(\mathrm{n}=183)$ & $92(50 \%)$ & $65(36 \%)$ & $26(14 \%)$ \\
\hline Recurrent $(\mathrm{n}=47)$ & $14(30 \%)$ & $21(45 \%)$ & $12(25 \%)$ \\
\hline
\end{tabular}

Conclusions: $\mathrm{H} 3 \mathrm{~K} 27 \mathrm{me} 3$ loss is more frequent among grade III and recurrent meningiomas. H3K27me3 loss is independently associated with worse OS. H3K27me3 loss is also associated with worse LRFS; however, this association is not independent of grade. Additional studies are required to identify the underlying mechanisms leading to $\mathrm{H} 3 \mathrm{~K} 27 \mathrm{me} 3$ loss in meningiomas.

1728 Primary Histiocytoid/signet Ring Cell Carcinoma of the Eyelid: Clinicopathologic Analysis with Evaluation of the E-cadherin/B-catenin Complex

Maria D Estopinal, Lavinia P Middleton, Michelle D Williams. MD Anderson Cancer Center, Houston, TX.

Background: Primary histiocytoid/signet ring cell carcinoma(SRCC) of the eyelid is a rare, aggressive neoplasm favored to arise from the eccrine sweat glands. Histologically, this tumor shows diffuse infiltration of the eyelid by single cells with histiocytoid appearance resembling SRCC of the gastrointestinal tract and breast. In SRCC of gastric and breast origin, E-cadherin dysregulation plays a central role in pathogenesis. Specifically, E-cadherin/B-catenin complex is required to maintain the integrity of epithelial cell-cell contact and keeps Wnt/B-catenin signals in check. Additionally, during tumor progression, epithelial-mesenchymal transition(EMT) alters E-cadherin expression with elevated SRC. As mechanisms contributing to tumorigenesis of histiocytoid/SRCC of the eyelid remain unknown, we analyze protein expression in key genes in E-cadherin related pathways.

Design: Three cases of primary histiocytoid/SRCC of the eyelid and orbit were treated at MD Anderson Cancer Center with surgical resection from 1990-2016. Clinicopathologic findings were evaluated and confirmed the primary site of origin. Immunohistochemical studies for expression of E-cadherin, B-catenin, c-Myc, cyclin D1, SRC, p53 were performed.

Results: We report 3 eyelid tumors that histologically proved to be histiocytoid/SRCC in 3 male patients aged 55-81 years. Clinically, infiltrative, firm cutaneous masses encircling the periocular area and orbit are noted. One of the patients has history of contralateral eyelid involvement, status post radiotherapy. Microscopically, cords of atypical histiocytoid cells arising from eccrine dermal ducts are identified infiltrating skeletal muscle and orbit. Perineural invasion is seen. Follow-up examination shows evidence of metastatic disease to the neck in one of the patients. Immunohistochemically, E-cadherin expression is completely lost in 2 of 3 cases which is accompanied by heterogeneous/loss of B-catenin expression. Focal cyclin D1 is noted in all 3 cases. Conversely, one case retains E-cadherin with associated increased SRC compared to the other 2 tumors with weak SRC expression. Alterations in p53 and c-Myc expression are not identified.

Conclusions: Histologic findings continue to link histocytoid/SRCC of the eyelid with origin in the eccrine glands; however, metastasis to this region must be excluded. We believe this tumor is biologically similar to primary histiocytoid/SRCC of breast with alterations in expression of the E-cadherin/B-catenin complex. EMT may also contribute to pathogenesis and requires further evaluation.

1729 Mantle Cell Lymphoma (MCL) of Orbit and Ocular Adnexa: Clinicopathological Features and Diagnostic Utility of SOX11 Monoclonal Antibody Immunohistochemistry (IHC)

Mukul Divatia, Andreia Barbieri, Juan Ortiz, April Ewton, Patricia Chevez-Barrios. Houston Methodist Hospital, Houston, TX.

Background: MCL is a B-cell lymphoproliferative disorder (B-LPD) that involves the orbit and ocular adnexa with a more aggressive course than other small B-LPDs. MCL demonstrates the presence of $t(11 ; 14)(q 13 ; q 32)$ with overexpression of cyclin D1. Diagnosis depends upon IHC demonstration of cyclin D1 protein expression and translocation detection in cyclin-D1 negative cases. Variant morphologies, including blastoid and pleomorphic MCL, make the diagnosis challenging. Proper diagnosis of MCL is crucial to patient care as patients are often treated aggressively, including with stem cell transplantation.

SOX11, a member of the SOX family of transcription factors, plays a role in neural development. It has been detected in the majority of MCLs, including those with variant morphology and is negative in almost all other B-LPDs.

Design: We reviewed lymphoma cases involving the orbit and ocular adnexa diagnosed during 2007-16. MCL cases were reviewed for morphology, clinical parameters including age, gender, clinical features, and systemic involvement. IHC/flow cytometry results were documented.

SOX11 IHC was performed on formalin-fixed paraffin- embedded tissue in MCL cases using an automated immunostainer (Bond-Max, Leica Microsystems Inc.) and monoclonal anti-SOX11 antibody Cell Marque clone MRQ-58 (1:100 dilution). Specimens with $>10 \%$ nuclear staining were considered positive.
Results: Of the 8 MCL cases identified, there were 7 orbital and 1 conjunctival tumors. There were 6 males and 2 females; age range of 55-77 (mean: 67.5) years. Bilateral disease was not seen; 5 right-sided and 3 left-sided cases. Orbital/adnexal MCL as first presenting symptom comprised $75 \%$ of cases. Prior systemic involvement was seen in 2 cases with relapse. The most frequent symptoms were pain, epiphora and diplopia; most frequent clinical signs were eyelid mass, conjunctival swelling and proptosis. All patients received rituximab chemotherapy and 3 cases are in complete remission. On IHC/ flow cytometry, all cases expressed B-cell marker CD20 and cyclin D1. CD5 was positive in 7/8 cases. Kappa and lambda light chain restriction was seen in 2 and 6 cases respectively. Strong and diffuse SOX11 staining ( $>75 \%$ cells) was seen in all 8 cases.

Conclusions: The orbit and adnexal region is involved by MCL in elderly patients with a male predominance and can be the first presentation site. SOX11 IHC is a reliable tool to identify MCLs in small biopsies in conjunction with cyclin D1, including cases with variant immunophenotypes and/or morphology.

1730 GlioSeq Targeted NGS Panel Provides Accurate Genetic Profiling of Pediatric CNS Tumors for Clinical Care

Soufiane El Hallani, Ronald L Hamilton, Craig M Horbinski, Ian F Pollack, Abigail Wald, Yuri Nikiforov, Somak Roy, Marina N Nikiforova. University of Pittsburgh Medical Centre, Pittsburgh, PA; Northwestern Memorial Hospital, Chicago, IL.

Background: Genomic analyses of pediatric brain tumors have discovered a number of specific molecular alterations and signatures that, when combined with histology, can re-classify these tumors (PMID: 26304884) and improve patient management. The aim of this study was to evaluate the clinical utility of testing pediatric CNS tumors with our GlioSeq NGS panel, and assess whether the emerging research-based molecular classifications can be reproduced in the clinical setting.

Design: We reviewed pediatric and young adult CNS tumors tested in our institution with GlioSeq panel. GlioSeq is a targeted NGS panel designed to detect point mutations/ indels in 30 CNS tumors related genes, copy number variations (CNVs) in 24 genes, and 14 types of gene fusions using minimal nucleic acid material from clinical FFPE specimens (PMID: 26681766). Information on demographics, histopathological diagnosis, and reported genetic alterations were included in the study.

Results: A total of 84 CNS tumors were tested with GlioSeq, including 54 gliomas ( 21 grade I, 5 grade II, 15 grade III, and 13 grade IV) 16 neuroglial tumors (14 grade I, 2 grade III), 11 medulloblastomas, 2 atypical choroid plexus papilloma (grade II) and 1 PNET (grade IV). GlioSeq NGS panel detected one or more genetic alterations in 60 cases $(71 \%)$, of which $30(50 \%)$ modified the final diagnosis, as recommended by the 2016 WHO classification. A summary of the molecular findings are shown in figure 1 .

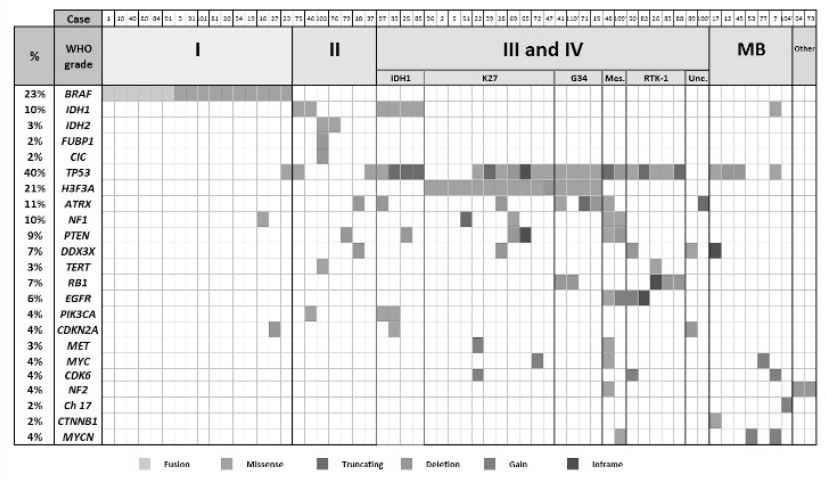

Based on detected genetic alterations, we were able to categorize 28 out of 30 high-grade gliomas (93\%) into K27, G34, IDH, RTK, or Mesenchymal subgroups of the newly proposed molecular classification of pediatric high grade gliomas.

Conclusions: In the majority of cases, molecular profiling of pediatric CNS tumors by GlioSeq enabled the detection of genetic alterations that enhanced diagnostic accuracy. It also correctly assigned the correct molecular subgroup in the vast majority of pediatric high-grade gliomas, providing valuable information for precision diagnosis and patient management.

\section{Expresion of HER2 in Ocular Surface Squamous Neoplasia} Evangelina Esposito, Pablo Zoroquiain, Ana B Toledo Dias, Miguel N Burnier. McGill University, Montreal, QC, Canada.

Background: C-erbB-2/neu (HER2) is an oncogene associated with poor prognosis, metastatic potential and aggressive biological behavior in several tumors including breast, stomach and others. HER2 amplification is also associated with the responders to anti-HER2 treatment. Ocular surface squamous neoplasia (OSSN) is a spectrum of neoplastic lesions of the conjunctiva ranging from papilloma to invasive squamous carcinoma (SCC). Moreover, SCC is the most frequent malignant neoplasia of the conjunctiva and $33 \%$ of them will metastasize. The aim of this study is to evaluate HER2 expression in the progression of OSSN and as a potential therapeutical target. Design: Ninety eight OSSN lesions including 17 papillomas (P), 27 conjunctival intraepithelial neoplasia (CIN) I, 19 CIN II, 13 CIN III and 11 SCC were evaluated. Eleven conjunctivas from eye bank eyes (NHE) were also included in the analysis. Immunohistochemistry was performed to analyze HER2 (clone 29D8) expression. HER2 expression was graded as follows: Complete membrane staining in more than $10 \%$ of malignant cells $(3+)$; week to moderate complete staining in more than $10 \%$ of malignant cells (2+); no or fewer than $10 \%$ cells staining $(0$ to $1+)$ respectively. Positive 
HER2 was considered when a score $2+$ or $3+$ was detected. Breast carcinoma with HER2 amplification was used as positive control. Statistical analysis was performed using Fischer's-Exact Test.

Results: HER 2 positive expression was detected in $6 \%$ of $\mathrm{P}(100 \%$ score $2+), 11 \%$ of CIN I ( $100 \%$ score $2+), 32 \%$ of CIN II ( $100 \%$ score $2+), 30 \%$ of CIN III ( $100 \%$ score $2+), 29 \%$ of SCC $(50 \%$ score $2+$ and $50 \%$ score $3+$ ). Benign lesions showed a lower HER2 expression than in situ $(\mathrm{p}=0.03)$ and invasive tumors $(\mathrm{p}=0.02)$ Moreover, no correlation between HER2 and age or tumor thickness in SCC was found ( $\mathrm{p}>0.05)$. Conclusions: HER2 is expressed in conjunctival OSSN. Moreover, one third of the invasive cases were graded as $2+$ or $3+$. These results support the evaluation of antiHER2 therapy in conjunctival SCC. Pre-clinical trials should be performed to determine the importance of this marker in conjunctival SCC for predicting response to treatments targeting this pathway.

RMDO-2, Olig-2 and Synaptophysin Expression Is a Frequent Event in Malignant Melanoma: Diagnostic Pitfalls in Glial Tumor

Taliya Farooq, Esther C Yoon, Anas Mashlah, Sina Zomorrodian, George Kleinman. New York Medical College at Westchester Medical Center, Valhalla, NY.

Background: Malignant melanomas (MM) are known to express S-100, HMB-45 and Mart-1 and vimentin among intermediate filaments. RMDO-2 (non- phosphorylated Neurofilament, NF) expression by MM has never been reported in literature and this phenomenon is not well-known. The diagnosis of MM can be challenging because melanoma is notorious for mimicking other tumors. Immunopositivity in MM fo antigens associated with other tumors may further obscure the diagnosis, particularly when there is no history of previous melanoma. The purpose of this study is to determine the frequency of immunopositivity for antigens associated with primary central nervous system tumors; RMDO-2 protein (non-phosphorylated NF), CD 56, synaptophysin and Olig-2 in MM.

Design: Twenty-four cases MM from Brain $(n=14)$, MM other sites, $(n=10)$ were retrieved from Westchester Medical Center, valhalla, NY; including cases from 19 males and 5 Females. Prior diagnoses were confirmed by re-review of hematoxylin and eosin sections and relevant (e.g., S100 protein, HMB45 and Melan-A) IHC studies. Sections were subjected for IHC stain for CD56 (Cell Marque, 1:100 dilution), RMDO2 (Sigma, 1:100 dilution), synaptophysin (Ventana, 1:100 dilution) and Olig-2 (Cell Marque, 1:100 dilution).

Results: 1) All cases were positive for $\mathrm{S} 100$ and at least one of the melanocytic markers (e.g HMB-45 or Melan-A). IHC results for other antigens are shown in table below:

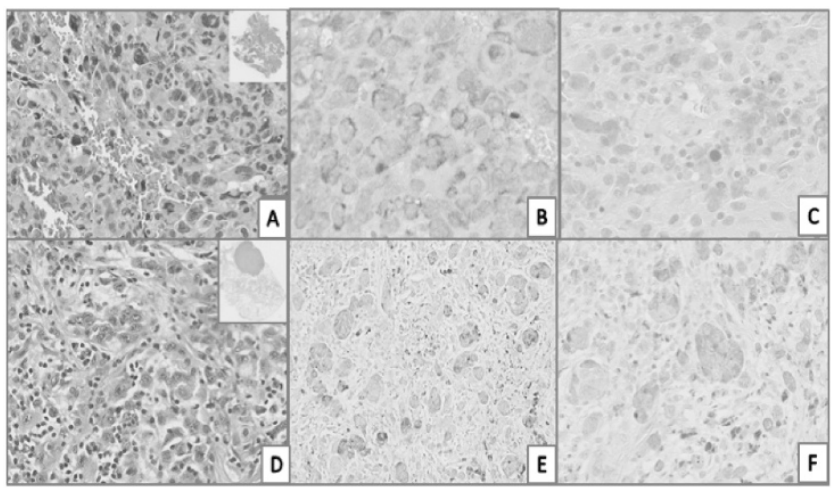

A) H\&E, B) RMDO-2, C) Synaptophysin in MM to Brain are positive, D) H\&E, E) RMDO-2 and F) Olig-2 from MM Lung.

\begin{tabular}{|l|l|l|l|}
\hline Antigen & MM to Brain & MM to Other Sites & Total Cases \\
\hline S100 protein & $13 / 14(93 \%)$ & $7 / 10(70 \%)$ & $(20 / 24) 83 \%$ \\
\hline RMDO-2 & $13 / 14(93 \%)$ & $1 / 10(10 \%)$ & $(14 / 24) 58 \%$ \\
\hline Olig-2 & $1 / 14(7 \%)$ & $4 / 10(40 \%)$ & $(5 / 24) 21 \%$ \\
\hline Synaptophysin & $3 / 14(21 \%)$ & $4 / 10(40 \%)$ & $(7 / 24) 29 \%$ \\
\hline CD-56 & $2 / 14(14 \%)$ & $5 / 10(50 \%)$ & $(7 / 24) 29 \%$ \\
\hline
\end{tabular}

Conclusions: 1) Heterogeneous expression of RMDO-2, Olig-2 and synaptophysin was found in significant subsets of MM in brain, representing potentially serious diagnostic pitfalls. 2) MM showing anomalous RMDO-2 and synaptophysin expression may easily be mistaken for primary CNS gliomas and neuroendocrine tumors. Awareness of this phenomenon and an appropriate melanocytic iHC panel should facilitate the diagnosis of metastatic melanoma with unusual immunophenotypes.

\section{CSF Cytokine Profiles Discriminate CNS B-Cell Lymphoma} from Other Neuropathologic Processes

Danielle Fortuna, Amity L Roberts, Larry A Harshyne, D Craig Hooper, Mark T Curtis. Thomas Jefferson University Hospital, Philadelphia, PA.

Background: Primary CNS lymphoma is an aggressive disease, accounting for up to $3 \%$ of primary brain tumors and is comprised mostly of B-cell lymphomas (BCL). Whereas immunodeficient individuals are at increased risk, primary BCL (PBCL) is increasing in the immunocompetent, especially the elderly. Differential diagnosis of $\mathrm{BCL}$, based on imaging and symptoms, is broad and includes gliomas, infection, and autoimmune disease. Given its frequent parenchymal location, CSF flow cytometry and cytology often yield false-negative results. We examined CSF cytokine levels in various CNS diseases in order to identify clinically useful profiles distinguishing BCL from clinical mimics.
Design: CSF levels (pg/ml) of 42 cytokines were measured using a bead based ELISA in the following samples: 4 PBCL, 2 systemic BCL (SBCL), 3 malignant gliomas (T), 12 CNS infections (I), 7 autoimmune diseases (A), 4 multiple sclerosis (MS), 3 controls (C). Agglomerative hierarchical clustering (AHC) with heatmap was performed. Mann-Whitney test for significance was used to compare groups. Receiver operator characteristic (ROC) curve was used to determine the utility of analytes as tests.

Results: AHC depicts three major clusters in which all PBCL, SBCL, and autoimmune/ demyelinating diseases form a discrete class.

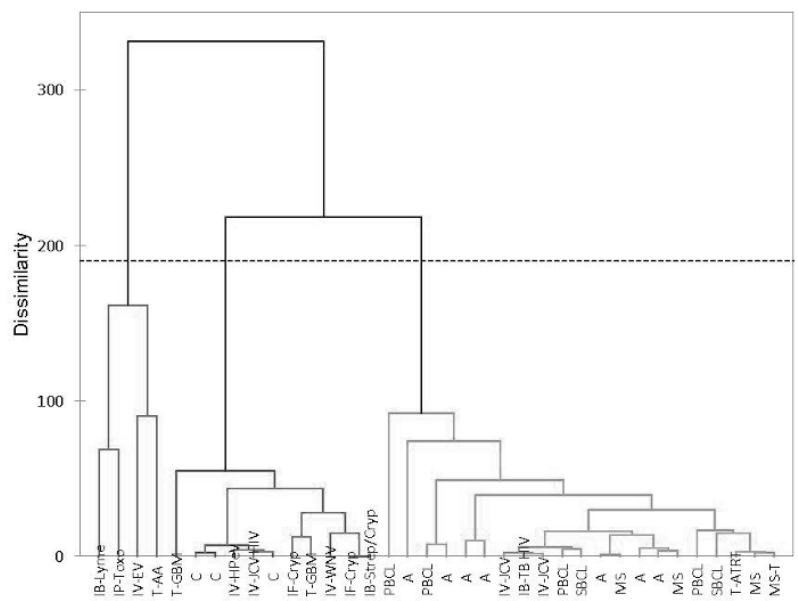

The heatmap demonstrated the following class properties: elevated IL1b, IL2, IL3, IL4, IL5, TNF $\beta$, IL12p70; lower levels of IFN $\gamma$, MIP1a, IL1Ra, sCD40L. This pattern directly contrasts expression seen in the infection and tumor classes. PDGF-AA can discriminate lymphoma (PBCL/SBCL) from autoimmune disease ( $\mathrm{p}=0.009$ by MannWhitney; $\mathrm{AUC}=0.853$ by ROC) with higher levels in lymphoma.

Conclusions: We have shown a robust characterization of CNS diseases based on cytokine expression, which holds clinical potential. When adjunct testing (cytology and flow cytometry) is non-informative, $\mathrm{BCL}$ can be distinguished from its clinical mimics based on cytokine profiling. PDGF-AA is a useful discriminating analyte, such that PDGF-AA is significantly higher in lymphoma compared to other diseases within its cytokine-based class. Given the observed clustering, our study has also highlights that lymphoma and autoimmune/demyelinating diseases may share similar, pathogenic properties related to disease genesis.

1734 The Role of Histopathologic Subtype in the Setting of Hippocampal Sclerosis-Associated Mesial Temporal Lobe Epilepsy Jordan M Gales, Richard A Prayson. Cleveland Clinic Lerner College of Medicine, Cleveland, $\mathrm{OH}$; Cleveland Clinic, Cleveland, $\mathrm{OH}$

Background: Hippocampal sclerosis (HS) and focal cortical dysplasia are among the most common findings in those undergoing surgery for refractory mesial temporal lobe epilepsy (MTLE). Existing data regarding differences among the most recent International League Against Epilepsy (ILAE) HS subtypes remains limited. Focal cortical dysplasia of the adjacent temporal lobe is increasingly reported to coexist with HS. The relationship between the two remains unclear. This study sought to examine the clinical significance of HS subtype and coexistent focal cortical dysplasia among those undergoing epilepsy surgery for MTLE.

Design: 307 resections for temporal lobe epilepsy with ipsilateral HS were reviewed (mean age $\pm \mathrm{SD}, 37 \pm 15$ years, $56 \%$ women). HS and coexistent lesions of focal cortica dysplasia were classified according to ILAE consensus guidelines. Medical records were reviewed for data on seizure recurrence and seizure burden during clinical follow-up (mean duration $\pm \mathrm{SD}, 5 \pm 4$ years)

Results: Cases of typical HS (ILAE type I) predominated [ILAE type Ia: $41 \%$, Ib: 47\%, II: $11 \%$, III: $0.7 \%$ ]. The HS subtypes shared similar demographic and etiologic characteristics, as well as associated pathology and post-operative seizure outcomes at 2 and 5 years following surgery. Individuals with type Ib HS were more likely to remain seizure free at long term follow-up when compared to other subtypes, and they reported a later age of seizure onset. Two hundred-forty three cases (79\%) demonstrated focal cortical dysplasia within the adjacent temporal lobe. Its presence was associated with a significantly decreased risk of seizure recurrence $(p=.02)$, especially during the early post-operative-period. When present, focal cortical dysplasia was predominantly Type I (98\%). The effect of coexistent focal cortical dysplasia upon overall epilepsy surgery outcomes at 2 years, 5 years, and long term follow-up was not significant, when measured by the Engel epilepsy surgery outcome scale.

Conclusions: HS subtype does not appear to affect epilepsy surgery outcomes, despite some clinical differences between the subgroups. Focal cortical dysplasia is often observed in association with HS in MTLE; the clinical implications of this remain uncertain. However, the findings of this study suggest its presence is associated with better post-operative outcomes when compared to those with isolated HS-associated MTLE. 
1735 New Hypothesis in the Pathogenesis of Diabetic Retinopathy Alireza Ghaffarieh. Indiana University, Indianapolis, IN.

Background: DIABETIC RETINOPATHY, A retinal vascular disorder that occurs as a complication of diabetes mellitus (DM), is a leading cause of blindness in the United States.

Although brain and retina are embryologically similar, and both vascular beds are exposed to similar concentrations of blood glucose, the extensive microvascular pathology develops only in the retinal capillaries, not in the cerebral capillaries. The initial step in the delivery of glucose to the retina and brain is its transport across the blood- retinal barrier (BRB) or the blood-brain barrier (BBB) by the major glucose transporter, GLUT-1. Glucose influx in retina exceeded that of the cortex by about threefold for the nondiabetic and the glucose levels in the diabetic retina are fourfold to sixfold greater than the nondiabetic retina. The investigators concluded that the expression of GLUT-1 in the microvasculature does not account for the glucose accumulation in the retina in diabetes.

G6Pase is found mainly in the liver and the kidney, plays a key role in the homeostatic regulation. We hypothesized that the increase in retinal glucose levels during diabetes is from increased entry into retina at the RPE-choroid interface through glucose-6phosphatase (G6P)-mediated activity of retinal pigment epithelium cells

Design: For immunostaining, the sections eye from patients with advanced stage diabetes and normal were deparaffinized, rehydrated and anti-glucose-6- phosphatase antibody has been applied. We also treated diabetic mice with intravitreal injection of glucose-6- phosphatase inhibitor.

Results: For the first time, We have detected the glucose-6- phosphatase enzyme activity in the human retinal pigment epithelium. After blocking this enzyme in the diabetic mice model ,the severity of diabetic retinopathy was decreased.
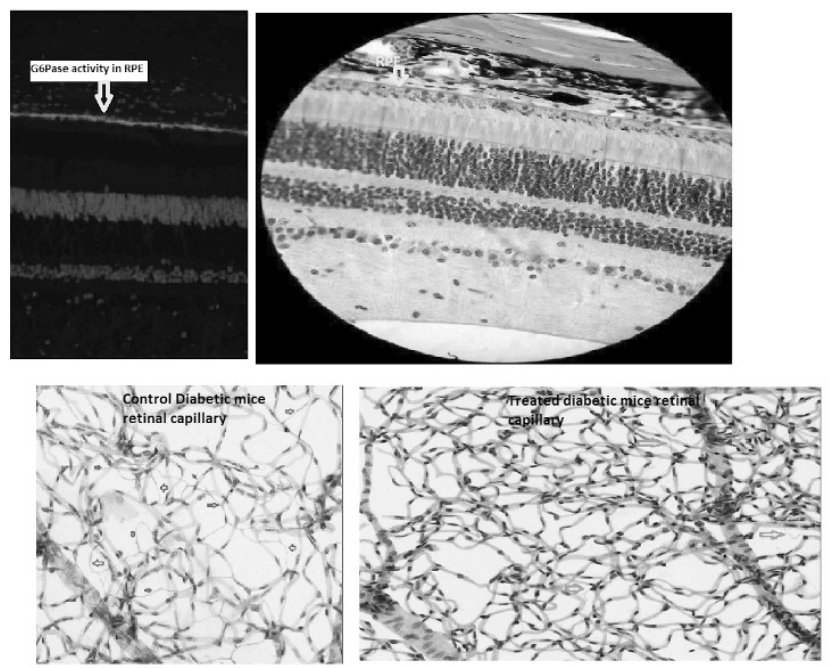

Conclusions: In Diabetes, the large amount of glucose enters the retina via the retinal pigment epithelium and these estimates are consistent with the presence of appreciable amounts of Glucose-6-Phosphatase. Blocking the glucose-6- phosphatase could be one of the new modalities of prevention and treatment for diabetic retinopathy.

\section{Determining IDH Status in Gliomas Using IDH1 R132H Antibody} and Polymerase Chain Reaction

Dibson D Gondim, Melissa AH Gener, Liang Cheng, Eyas M Hattab. Indiana University, Indianapolis, IN; University of Louisville, Louisville, KY; Children's Mercy Hospital, Kansas City, MO

Background: Determination of the mutation status of the IDH (isocitrate dehydrogenase) gene in diffuse gliomas has not only become the standard of care, but is also now incorporated in the nomenclature of main entities of this group, beginning with the WHO 2016 Classification of Tumors of the CNS. IDH mutation status has prognostic, therapeutic, and diagnostic relevance. Among the several methodologies available for studying IDH mutations, assessment by IHC (immunohistochemistry) has the advantage of being incorporated in the routine pathology workflow and has the potential of detecting $\sim 90 \%$ percent of the mutations. The WHO 2016 publication suggests confirmation of negative IHC results by another diagnostic modality in all grades II and III diffuse gliomas or in grade IV glial tumors if the patient is younger than 55 years. To evaluate the WHO guidelines, we compared the detection of IDH mutation by IHC and PCR.

Design: Monoclonal antibody for IDH1 R132H (Dianova-clone H09) and IDH1/2 RGQ PCR Kit (Qiagen) were both used to determine IDH mutation status. We included 62 cases of both diffuse and circumscribed gliomas ( 31 males, 31 females). The tumors were comprised of 30 glioblastomas (GBM), 11 grade III diffuse gliomas ( 6 anaplastic astrocytomas, 2 oligodendroglioma, 3 oligoastrocytomas), 17 grade II diffuse gliomas (9 diffuse astrocytomas, 4 oligodendrogliomas, 4 oligoastrocytomas), 1 anaplastic pilocytic astrocytoma, 2 pilocytic astrocytomas, and 1 ganglioglioma.

Results: Of the 62 cases, 12 had IDH mutation by IHC and 15 by PCR; 3 false-negative results by IHC were identified. PCR detected IDH1 R132H (11 cases); IDH1 R132C (1 case); IDH2 R172, NOS (1 case); IDH1 R132, NOS (1 case); and IDH2 R172K (1 case). R132H antibody had high specificity $(100 \%)$ and sensitivity $(80 \%)$ to detect IDH mutations. More importantly, the positive predictive value was $100 \%$. The three false-negative cases were two anaplastic astrocytomas (patients were 38 and 35 years of ages) and one diffuse astrocytoma (33 years of age). IHC did not detected IDH1 R132C and IDH2 R172 mutations, as expected. The antibody detected 12/13 IDH1 R132H mutations. Among the IDH-wildtype diffuse gliomas, GBMs had a highest proportion of wild-type cases ( $93 \%$ ).

Conclusions: Evaluation of IDH mutation could be approached in a stepwise fashion. The high positive predictive value of IDH1 R132H IHC justifies ordering it as the first step of evaluation. Nonimmunoreactive cases could then be studied by PCR following recommendations of $2016 \mathrm{WHO}$

1737 Utility of Somatostatin Receptor 2a Immunohistochemistry in the Clear Cell Tumor Differential of the Central Nervous System

Abigail L Goodman, Jose E Velazquez Vega, Stewart Neill, Matthew Schniederjan, Stephen Hunter, Cynthia Cohen, Daniel J Brat. Emory University Hospital, Atlanta, GA. Background: Histologically similar primary and metastatic clear cell tumors occasionally involve the central nervous system (CNS) and can be difficult to distinguish by morphology. This is especially true for tumors with clear, often vacuolated cytoplasm and a rich vascular network, including metastatic clear cell renal cell carcinoma (mCCRCC), hemangioblastoma, and microcystic and clear cell meningiomas. We evaluated an immunohistochemical panel that incorporates somatostatin receptor $2 \mathrm{a}$ (SSTR2a) for distinguishing entities within the differential of clear cell CNS tumors. Design: We performed a search in our institutional database for cases of mCCRCC, hemangioblastoma, and clear cell and microcystic meningiomas. An immunohistochemical panel that included SSTR2a (UMB1, Abcam, Cambridge, CA 1:400, Leica), CA IX, Pax 8, and Inhibin was performed on all cases. Criteria for positivity were established for each marker and a consensus was achieved for positive and negative staining for each tumor and stain

Results: We included 62 cases: 18 mCCRCCs, 30 hemangioblastomas, 3 clear cell meningiomas and 11 microcystic meningiomas. SSTR2a was strong and diffusely positive in 12 of the 14 meningiomas, but was negative in all mCCRCC or hemangioblastomas ( $100 \%$ specificity, $86 \%$ sensitivity in this study). CA IX labeled all mCCRCCs and hemangioblastomas strongly and diffusely but was also positive in 9 microcystic meningiomas and 1 clear cell meningioma. All cases of mCCRCC demonstrated strong nuclear positivity for Pax 8 in at least two-thirds of the cells; no immunoreactivity was observed in any other tumor type $(100 \%$ sensitivity and specificity). Inhibin had high sensitivity (87\%) and specificity (97\%) for hemangioblastomas, although staining was frequently focal and weak.

\begin{tabular}{|c|c|c|c|c|}
\hline Tumor Type (n=62) & SSTR2a (\%) & CA IX (\%) & Pax 8 (\%) & Inhibin (\%) \\
\hline mCCRCC (18) & 0 & $18(100)$ & $18(100)$ & $1(6)$ \\
\hline Hemangioblastoma (30) & 0 & $30(100)$ & 0 & $26(87)$ \\
\hline Clear Cell Meningioma (3) & $2(67)$ & $1(33)$ & 0 & 0 \\
\hline Microcystic Meningioma (11) & $10(91)$ & $9(82)$ & 0 & 0 \\
\hline
\end{tabular}

Conclusions: The histopathologic distinction of CNS clear cell neoplasms is greatly aided by the use of an immunohistochemical panel that includes SST2Ra, Pax8 and inhibin. SSTR2a immunoreactivity was highly sensitive and specific for clear cell meningioma variants, while Pax8 and inhibin were sensitive and specific for mCCRCC and hemangioblastoma, respectively. CA IX labeled all mCCRCCs and hemangioblastomas strongly and diffusely, however it was frequently positive in meningiomas.

\section{Analysis of Immunobiologic Markers in Primary and Recurrent} Glioblastoma

Wesley Hiser, Paul Kubilis, Duane Mitchell, Jesse L Kresak. University of Florida, Gainesville, FL

Background: Glioblastoma (GBM) remains an invariably fatal disease and tumor progression may be linked to immune evasion strategies. Immune system function and tumor suppression relies on the complex interplay between stimulatory and inhibitory signals present in the tumoral immune microenvironment. GBMs generate a varied immune response and understanding the immune microenvironment may lead to novel immunotherapy treatments modalities. The goal of this study was to evaluate the expression of immunologic markers of potential clinical significance in primary versus recurrent GBMs and assess the relationship between these markers and molecular characteristics of GBMs.

Design: Fifty cases of GBM (38 primary and 12 recurrent) were evaluated and analyzed with immunohistochemistry for multiple immunobiologic markers (CD3, CD8, FoxP3, CD68, CD163, PD1, PDL1, CTLA4, CD70). Included were four paired cases of primary and recurrent GBM. Immunoreactivity was analyzed using Aperio software. In order to avoid nonspecific background reactivity, analysis was limited to only tumor-rich regions. Additionally, the software was calibrated to only recognize strongly positive immunoreactivity. Degree of strong positive immunoreactivity within the tumor was compared to patient and tumor characteristics including age, gender, MGMT promoter methylation status, and ATRX, p53, and IDH1 mutation status.

Results: Using odds ratios, no significant difference was identified in any immune marker between the primary and recurrent glioblastomas, nor was a significant change in immunoreactivity identified among age intervals. IDH1 mutated GBMs had statistically significant decreased expression of CD163 and CD70 and a trend for decreased PD1, CTLA4, and Foxp3. ATRX-mutated GBMs exhibited statistically significant increased CD3 immunoreactivity, while those with p53 mutations were found to have significantly increased CTLA4 immunoreactivity. The odds of having strong CD8 and CD68 reactivity was significantly less in MGMT methylated tumors. No trends were identified amongst the paired specimens. Overall, the ratios of CD8, foxp3, and CTLA4 to CD3 and of $\mathrm{CD} 168$ to $\mathrm{CD} 68$ decreased between primary and recurrent tumors. 
Conclusions: GBMs with features associated with a better prognosis (IDH1 mutation) exhibited an immunophenotype consistent with anti-tumor effects. The findings also suggest that the immunobiologic markers have greater association with the molecular characteristics of the tumor than with primary/recurrent status or age.

1739 CD163 and PD1-PDL1 Promote a Hostile Microenvironment fo CD8 Lymphocytes and Predict Poorer Survival in Glioblastoma

Miguel A Idoate, Alvaro Lopez Janeiro, Arturo Lecumberri, Iñigo Arana, Ricardo DiezValle, Francisco Guillen, Luis Mejias. University of Navarra, Pamplona, Navarra, Spain. Background: The immune response against Glioblastomas (GB) depends on inhibitory and activating forces. Programmed death ligand 1 (PDL1), its corresponding T cell receptor (PD1) and M2 macrophages are postulated to be relevant inhibitory mechanisms. CD137 expression is a relevant lymphocyte activation marker. In addition, it is important to standardize and quantify the expression of these factors.

Design: Seventy-six clinically and molecularly characterized primary GB were studied. Immunohistochemical study against CD8 (clone C8/144B), PD1 (clone NAT105), PDL1 (clones SP142 and 28.8), CD137 (clone BBK-2) and CD163 (clone MRQ-26, M2 macrophages) was obtained. We evaluated regions of interest in two ways: a semiquantitative assessment by three observers and a quantitative one using digital image analysis. Statistical analyses were performed using STATA (version 12.0).

Results: PDL1 clone 28.8 was mostly membranous and present in $30 \%$ of GB. Clone SP142 was cytoplasmic and present in $47 \%$ of GB. Concordance between both clones was poor $(\mathrm{Kappa}=0.33, \mathrm{p}<0.001)$. We found good correlation between semiquantitative and quantitative evaluations for CD163, CD8 and PDL1 ( $\mathrm{p}<0.001)$. CD137 expression was extremely low ( $<1 \%$ in all specimens). No difference in overall survival (OS) between high and low grades of CD137 expression was found. Adjusted Cox model showed an association between CD163 (HR=1.04, $\mathrm{p}<0.05)$ and, surprisingly, CD8 $(\mathrm{HR}=1.06, \mathrm{p}<0.05)$, with a poorer OS. Higher CD8 values were associated with higher $\mathrm{M} 2$ macrophage infiltration $(\mathrm{R}=0.46)$. High $\mathrm{PD} 1$ expression was associated with poorer OS $(\mathrm{HR}=2.79, \mathrm{p}<0.001)$. Adjusted regression model showed no association between PDL1 expression and OS

Conclusions: PDL1 clone 28.8 is an adequate monoclonal antibody to determine PDL1 expression in GB. We propose the use of image analysis as a valid quantification approach. CD163 and PD1 immunohistochemical expression are associated with poorer prognosis in GB. We hypothesize that M2 macrophage (CD163) recruitment and PDL1 expression may be a defensive strategy upregulated by GB in response to tumor infiltrating lymphocytes. This is also coherent with the absence of CD137 immunostimulatory marker of CD8 lymphocytes.

\section{Expression of Sonic Hedgehog (SHH) Signaling Molecules in} Retinoblastoma and Medulloblastoma

Nafiseh Janaki, Amad Awadallah, Mark Cohen, Marta E Couce. University Hospitals Cleveland Medical Center, Cleveland, $\mathrm{OH}$.

Background: Retinoblastoma is the most common intraocular malignancy in children. Recent findings demonstrate an intricate genetic and epigenetic complexity behind these tumors following in most cases $R B 1$ inactivation. Better understanding of the pathways involved will likely help in the development of new therapeutic approaches for these patients. A recent study has demonstrated that SHH pathway activation in retinoblastoma is associated with advanced disease. SHH activation also underlies approximately one third of cases of medulloblastoma, a relatively common pediatric brain tumor. Pathological activation of SHH pathway is felt to be responsible for all desmoplastic medulloblastomas as well as some medulloblastomas with classic histology. Several antibodies to $\mathrm{SHH}$ signaling proteins are commercially available. Currently immunohistochemistry for Grb2-associated adaptor protein-1 (GAB1) is considered one of the best methods to infer SHH pathway activation in medulloblastomas.

We examined the expression profile of different $\mathrm{SHH}$ signaling proteins in retinoblastoma and medulloblastoma cases. We correlated these findings with clinicopathological parameters in order to identify the optimal immunohistochemical paradigm for assessing SHH pathway activation in these tumors.

Design: Tissue microarrays (TMA) were constructed from 11 retinoblastomas and 8 medulloblastomas, each containing duplicate tissue cores of 1 or $1.5 \mathrm{~mm}$ diameter sampled from the most representative areas of the donor blocks. Immunohistochemistry was performed using a BenchMark Ultra immunostainer (Ventana system). The following antibodies were used: SHH, GAB-1, GLI-1, GLI-2 and GLI-3. The results were considered positive if $10 \%$ or more of the tumor cells were positive. Semiquantitative evaluation of positive markers was also performed.

Results: As expected, all desmoplastic medulloblastomas and some classic medulloblastomas were positive with anti-GAB1 antibodies, with a moderate to high level of immunoreactivy. These cases also demonstrated predominantly high immunoreaction with $\mathrm{SHH}$ antibodies. Nine retinoblastoma cases were positive for anti$\mathrm{SHH}$, six of which were strongly positive. None of these cases were immunoreactive with GAB-1.

Conclusions: GAB-1 is the current surrogate marker of choice for SHH activity in medulloblastoma, although SHH immunostaining provides similarly reliable results. On the other hand, GAB-1 should not be considered a reliable marker with which to assess $\mathrm{SHH}$-activation in retinoblastomas.
1741 Loss of Expression of SWI/SNF Chromatin Remodeling Complex Proteins in Sporadic Pituitary Adenomas

Upasana Joneja, Markku M Miettinen, Varun Kshettry, James J Evans, Mark T Curtis. Thomas Jefferson University Hospital, Philadelphia, PA; National Cancer Institute Bethesda, MD.

Background: Pituitary adenomas account for $15 \%$ of all intra-cranial tumors. While considered benign, a high number of pituitary adenomas cause significant morbidity $5 \%$ of the pituitary adenomas are caused by germline mutations but only few genetic alterations have been described in sporadic pituitary adenomas suggesting epigenetic alterations may play a role. Chromatin remodeling proteins including the SWI/SNF complexes are involved in gene expression, and dysregulation of this system is known to cause a variety of tumors. In this study, we analyzed the expression of a number of $\mathrm{SWI} / \mathrm{SNF}$ complex proteins in sporadic pituitary adenomas to determine if their loss of expression may play a role in the biology of these tumors.

Design: Immunohistochemistry was performed on tissue microarrays of 154 pituitary adenomas to determine the expression of the SWI/SNF complex proteins INI-1, ARID1A, BRG1, and SMARCD3. Complete absence of staining was considered loss of expression. Additional markers associated with pituitary adenoma behavior including $\mathrm{Ki}-67, \mathrm{p} 53$ and IGF-1R were analyzed as were the mRNA regulator protein-HuR, an indicator of DNA methylation status- 5-hydroxymethylcytosine (5HMC), and tumor associated macrophage marker- CD163. The expression of all the SWI/SNF chromatin remodeling proteins was correlated with staining of the other selected markers and with tumor functionality, invasiveness and recurrence rate.

Results: Complete loss of expression was identified in $19 \%$ of the sporadic pituitary adenomas ( $10 \%$ for BRG1, $5 \%$ for ARID1A, 3\% for SMARCD3 and less than $1 \%$ for INI-1).Two cases showed concomitant loss of expression of ARID1A and BRG1 and two others showed concomitant loss of expression of BRG1 and SMARCD3. One case showed concomitant loss of BRG1, ARID1A and SMARCD3. No correlation was identified between loss of expression of SWI/SNF complex proteins and tumor functionality, invasiveness, recurrence or immunohistochemical staining for Ki67, p53, IGF-1R, HuR, 5-HMC, or CD163.

Conclusions: SWI/SNF chromatin remodeling protein expression is lost in $19 \%$ of sporadic pituitary adenomas. Dysregulation of the remodeling complexes may play a role in the genesis or progression of pituitary adenomas, reversal of which may provide a future therapeutic strategy for these tumors.

1742 Targeted Mutation Profiling by the Extended Next GenerationSequencing Assay and Clinical Utility of Actionable Genomic Alterations in CNS Tumors: A Single Academic Institution Experience Jong Kim, Kimberly J Newsom, Ashley P Ghiaseddin, Jesse L Kresak, Petr Starostik. University of Florida Health, Gainesville, FL.

Background: The use of targeted mutation profiling (TMP) is becoming an integral part of personalized management of patients with malignant tumors. Detecting a tumor's underlying genetic alteration (GA) can provide valuable diagnostic and prognostic information, yet the clinical actionability of TMP in brain tumors is unclear. The purpose of this study is to investigate if the use of TMP leads to implementation of genomically guided therapy in patients with difficult to treat brain tumors at our academic institution. Design: Our pathology archive was queried for CNS tumors which had been evaluated by extended next generation sequencing (NGS) assays. A total of 40 such CNS tumors were identified; 21 of which were evaluated using our in-house clinically validated NGS assay on the Ion Torrent Personal Genome Machine with the AmpliSeq Cancer Hotspot Panel v2 (reporting 33 genes) and 19 cases had been referred for Foundation One (FO) testing (Foundation Medicine, Cambridge, MA). A retrospective chart review was performed to identify therapies implemented in these patients.

Results: A total of 25 metastatic CNS tumors were analyzed by NGS ( 21 utilizing our in-house assay and 4 sequenced by $\mathrm{FO}$ ) and 15 primary CNS tumors were analyzed by FO. The FO platform revealed a total of $81 \mathrm{GA}(4.25 \mathrm{GA}$ and 1.47 actionable GA per each tumor). The in-house NGS assay revealed a total of $22 \mathrm{GA}(1.05 \mathrm{GA}$ and 0.09 actionable GA per tumor). The most commonly detected GA were TERT, CDKN2, TP53, NF1, EGFR, FGR, PTEN, ATRX, and KRAS. 19 out of 41 cases $(46 \%)$ harbored actionable GA. 3 of these patients received genomically-targeted therapy; one targeting AKT2 amplification in an anaplastic meningioma (FO) and 2 targeting EGFR in metastatic tumors (in-house assay)

Conclusions: $46 \%$ (19/41) of the tumors analyzed harbored actionable GA, however, only $16.7 \%$ (3/19) of patients received targeted therapy. Barriers to implementing targeted therapy are multifactorial. FDA approved targeted therapies are often reserved as 2nd line treatment to be used as the data on the efficacy of these drugs in the CNS is limited. In addition, access to the medication can be a time-consuming hurdle. Addressing key factors that facilitate the use of NGS results in oncologic care could benefit patients with difficult to treat CNS tumors.

1743 H3 K27M Mutation in Gangliogliomas Can Be Associated with Poor Prognosis

Bette Kleinschmidt-DeMasters, Andrew Donson, Nicholas Foreman, Kathleen Dorris. University of Colorado Anschutz Medical Campus, Aurora, CO; Children's Hospital Colorado, Aurora, CO

Background: Spinal cord and brainstem diffuse midline gliomas have been shown to have H3F3A (K27M) mutations, which are paralleled by presence of strong, diffuse nuclear immunoreactivity using a high fidelity immunohistochemistry (IHC) stain. Although a World Health Organization (WHO) grade of IV has been assigned to diffuse midline glioma, H3 K27M-mutant, it is becoming apparent that rarely low grade tumors, including several case reports of pilocytic astrocytoma and ganglioglioma (GG), may also show this mutation. In these examples, eventual tumor progression and demise was 
recorded. This suggests that the presence of mutation may portend adverse prognosis in any tumor type in which it occurs. Given our previous experience with midline GGs, both pediatric and adult, we re-interrogated all previously-published + new cases for H3 K27M by IHC. All had clinical followup and either BRAF V600E mutational or BRAF VE1 IHC testing.

Design: Review of files generated 14 patients with midline GGs (11 F : $3 \mathrm{M}), 7$ of which succumbed to their $\mathrm{GG}$, and 5 of which came to autopsy.

Results: 2 patients with midline GGs ( 1 brainstem: 1 spinal cord; $1 / 2$ were metastatic) were found to be $\mathrm{H} 3 \mathrm{~K} 27 \mathrm{M}$ mutant; both died of disease. The other 5 patients with demise were negative for mutation; $3 / 5$ were metastatic. 4 had $B R A F$ mutation; $1 / 4$ was metastatic. No case had both $\mathrm{H} 3 \mathrm{~K} 27 \mathrm{M}$ and BRAF V600E mutation. Neither mutant GG had unusual histological features.

Conclusions: H3 K27M mutation in GGs may be associated with poor prognosis, but the latter is not invariably associated with mutation.

\section{$1744 \quad$ Ultrastructural Examination of Skin Biopsies May Assist in Diagnosing Mitochondrial Cytopathy When Muscle Biopsies Yield Negative Results}

John L McAfee, Christine B Warren, Richard A Prayson. Cleveland Clinic Lerner College of Medicine, Cleveland, $\mathrm{OH}$; Cleveland Clinic, Cleveland, $\mathrm{OH}$.

Background: A recent consensus statement from the Mitochondrial Medicine Society includes a skeletal muscle and/or liver biopsy in its standard approach. The present study aims to investigate how frequently skin biopsies reveal mitochondrial abnormalities and to correlate skin findings with muscle biopsy results.

Design: A retrospective review 630 skin biopsies from 615 patients (1990-2015) that were evaluated for metabolic disease by electron microscopic (EM) examination. Of these patients, 178 also underwent a muscle biopsy.

Results: Of the 630 skin biopsies, 75 (12\%) showed EM abnormalities and 34 (5\%) specifically showed mitochondrial abnormalities including increased mitochondrial size $(n=27)$, reduced or abnormal cristae $(n=23)$, dense matrices $(n=20)$, and increased mitochondrial number $(\mathrm{n}=8)$. Sixteen samples demonstrated other findings including lysosomal abnormalities $(\mathrm{n}=13)$, lipid accumulation $(\mathrm{n}=2)$ or glycogen accumulation $(\mathrm{n}=1)$. Of the 34 patients with mitochondrial abnormalities on skin biopsy, 20 also had muscle biopsies performed; nine of these showed abnormalities specifically suggestive of a mitochondrial abnormality including absent cytochrome oxidase staining $(\mathrm{n}=2)$, increased subsarcolemmal NADH, SDH, or cytochrome oxidase staining $(\mathrm{n}=1)$, or EM findings including large mitochondrial size $(\mathrm{n}=5)$, abnormal mitochondrial structure $(\mathrm{n}=5)$, and increased mitochondrial number $(\mathrm{n}=4)$. The most common presenting symptoms were intellectual disability $(n=13)$, seizures $(n=12)$, encephalopathy $(n=9)$, and gastrointestinal disturbances $(\mathrm{n}=9)$. At last known follow-up, 12 patients had been given a definitive diagnosis of a mitochondrial disorder. Specific diagnoses include one patient each with Complex I deficiency, Complex III deficiency, Charcot-MarieTooth disease, pyruvate dehydrogenase deficiency, and Phelan-McDermid syndrome. Conclusions: There is a subset of patients for whom skin biopsy reveals mitochondrial abnormalities. Among these patients, $45 \%$ of muscle biopsies showed findings suggestive of a mitochondrial abnormality. A skin biopsy sometimes yields diagnostic clues suggestive of a mitochondrial cytopathy in cases with a negative muscle biopsy.

\section{Aggressive Genetic Signature Correlates with Tumor Cell} Proliferation in Supratentorial Ependymoma

Michael B Miller, Adrian Dubuc, Azra H Ligon, Keith L Ligon. Brigham and Women's Hospital, Boston, MA.

Background: Ependymomas comprise a class of glial neoplasms in the central nervous system. The W.H.O. 2016 current diagnostic guidelines specify grade II Ependymoma and grade III Anaplastic Ependymoma, but distinction between these is somewhat subjective, and definitive association with behavior has not been established. However, certain genetic variants, including C11 orf95-RELA fusion, CDKN2A loss, and 1q gain, have been reported as associated with poor outcomes. Major questions remain regarding the relationship between tumor genetics and histological features.

Design: In an effort to identify histological and genomic features which might improve ependymoma classification, we reviewed the histologic features, including mitotic rate and proliferation index (by MIB-1 immunohistochemistry), and genomic aberrations identified by microarray hybridization studies across a cohort of 40 patients, ages 2 - 70 years, who had a diagnosis of Ependymoma, Anaplastic Ependymoma, or Myxopapillary Ependymoma.

Results: Loss of the tumor suppressor gene CDKN2A, previously found to be associated with aggressive behavior, was observed in 8 cases, each of which was supratentorial and demonstrated a proliferation index greater than $15 \%$ (mean $26.2 \%$ ). The majority of cases with retained $\mathrm{CDKN} 2 \mathrm{~A}$ showed a proliferation index less than $15 \%$, particularly among supratentorial ependymomas. Among the few supratentorial cases which showed a proliferation index greater than $15 \%$ but retained CDKN2A, the deleterious chromosome 1q gain was found.

Conclusions: These findings suggest that a proliferation index of $>15 \%$ may identify a subset of supratentorial ependymomas with an aggressive genetic signature, bearing either CDKN2A loss or chromosome 1q gain. Infratentorial ependymomas (including posterior fossa and spinal cord sites) retained CDKN2A and demonstrated a wide range of proliferation indices.
1746 Unexpected Pathologic Findings in a Subset of Malformations of Cortical Development

Vera Paulson, Rebecca Folkerth, Sanda Alexandrescu. Boston Children's Hospital, Boston, MA; Harvard Medical School, Boston, MA.

Background: Imaging in chronic epilepsy may be indeterminate for neoplasia. We encountered an 8-year-old female with intractable epilepsy and imaging consistent with focal cortical dysplasia (FCD). The left temporal lobe and hippocampus were resected and showed changes of FCD. However, the presence of increased cellularity, mild glioneuronal atypia, and a positive BRAF V600E immunostain were consistent with ganglioglioma. This index case prompted a retrospective review of consecutive epilepsy specimens resected at Boston Children's Hospital (BCH) from 2012 to 2016 to determine the incidence of neoplasia in such specimens.

Design: We identified 121 patients with epileptic foci resections, and subsequently excluded from evaluation those with prior tumor diagnoses, tuberous sclerosis hemimegalencephaly, inflammatory processes, strokes, encephaloceles, and prior brain surgery. Demographic and clinical data, resection location, magnetic resonance imaging (MRI), and histological and immunohistochemical results (including a low grade glioma panel in 9 cases) were reviewed.

Results: Forty-five patients (19 male, 26 female) ranging in age from 7 months to 19 years old (median: 9 years) were identified. Their MRIs predominantly demonstrated malformations of cortical development, though the findings were indeterminate in 7 patients. There were 23 temporal lobe ( 13 with hippocampus), 18 frontal lobe, 3 parietal lobe, and 1 occipital lobe resection specimens. The specimens with non-neoplastic histology were classified according to the International League Against Epilepsy (ILAE) classification system as FCDIa (2), FCDIIa (17), FCDIIb (9), FCDIIIa (3), FCDIIId (1), mesial temporal sclerosis $(4,1$ with polymicrogyria), and polymicrogyria (1). Three cases demonstrated non-specific changes only. Five cases demonstrated increased cellularity and cytologic atypia and were classified as ganglioglioma (3), low grade glioma (1), and atypical glial proliferation (1) following immunohistochemistry and molecular studies. BRAF V600E mutation was present in all three gangliogliomas. Conclusions: Our study shows that $8.9 \%$ of BCH patients undergoing epilepsy surgery, without MRI studies definitive for tumor, have low grade glial and glioneuronal neoplasms. Therefore, the presence of increased cellularity and cytologic atypia, beyond the typical secondary reactive changes present in the setting of epilepsy, should prompt ancillary molecular studies for a neoplasm.

\section{Dutcher Bodies in Lacrimal Glands: Not Always a Reliable} Feature in Distinguishing Malignant from Benign Lymphoid Infiltrates

Sabrina Racine Brzostek, Jeffrey Ahlstedt, Yanhua Wang. Montefiore Medical Center/ Albert Einstein College of Medicine, Bronx, NY.

Background: Dutcher bodies are nuclear pseudoinclusions of eosinophilic PAS + cytoplasmic nuclear invaginations that have been used as clues in distinguishing a benign lymphoid aggregate from malignancy, such as multiple myeloma, lymphoplasmacytic lymphoma, and MALT lymphoma. This study describes a case series of 14 lacrimal gland surgical pathology cases wherein no lymphoproliferative disorder was identified, yet populations of Dutcher body containing plasma cells were frequently identified. Design: Montefiore Medical Center's LIS was data-mined using the keyword lacrimal gland during 4/2014 - 4/2016. Histology was reviewed, eliminating specimen lacking lacrimal glands. Clinical histories and $\mathrm{IHC}$ were reviewed for malignancy, presence of Dutcher bodies and IgG4 related disease. Additionally, 3 cases were selected to evaluate the Ig heavy chain profile of the plasma cells by IHC.

Results: The LIS search resulted in identifying 25 cases. Malignancy (small B cell lymphoma, marginal zone lymphoma and extranodal marginal zone lymphoma) had been reported in 3 cases; 2 cases were excluded due to lack of lacrimal glands within the specimen. Of the remaining 20 cases, 14 were identified as containing Dutcher bodies in the context of chronic dacryoadenititis, chronic inflammation or benign hyperplasia. Ig heavy chain staining on 3 of the benign specimens revealed a mixed, but predominate $\operatorname{IgA}+$, plasma cell population, when compared to a selected lymphoma with $\mathrm{IgG}^{+}$ plasma cells. B-cell rearrangement molecular testing on a Dutcher body-containing benign specimen also revealed polyclonality. IgG4 related disease had been evaluated in 7 of 14 benign Dutcher body cases, and found to be negative.

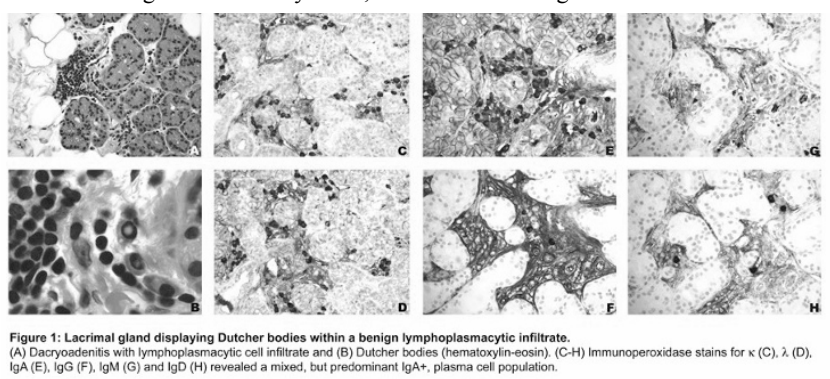

Conclusions: Historically Dutcher bodies have been associated with malignancy, with only 2 prior studies identifying the feature in benign conditions: chronic synovitis and ocular adnexal benign lymphoid hyperplasia. The findings in this study support the underappreciated association of Dutcher bodies in benign reactive conditions, such as dacryoadentitis. Therefore, caution must be exercised in utilizing Dutcher bodies in a low grade orbital lymphoma work up, especially in the lacrimal gland. 
1748 Is It Worthy to Reclassify Already Diagnosed Oligoastrocytomas in the Setting of the New WHO Criteria?

Irma Ramos-Oliver, Laura Abraira, Jordi Temprana-Salvador, Carolina Montecino, Jessica Camacho, Cristina Auger, Francisco Martínez-Ricarte, Santiago Ramon y Cajal, Elena Martínez-Sáez. Vall d'Hebron University Hospital, Barcelona, Spain.

Background: The new WHO classification of Central Nervous System tumors classifies morphologically mixed gliomas (classically diagnosed as oligoastrocytomas) into astrocytomas or oligodendrogliomas based on the preence of IDH and ATRX mutations, and $1 \mathrm{p} / 19 \mathrm{q}$ codeletion. The objective of this study was to apply the recent update of the WHO classificacion to reclassify recently diagnosed oligoastrocytomas $(\mathrm{OA})$ into oligodendrogliomas $(\mathrm{O})$, diffuse astrocytomas $(\mathrm{DA})$ or oligoastrocytomas $(\mathrm{OA})$ with a dual genotype. However, as the grading criteria for oligodendroglioma and astrocytomas are different, applying the new diagnostic algorithms could imply a change in the histological grade of previously diagnosed oligoastrocytomas.

Design: Twenty-six cases of OA, 19 grade II and 7 grade III, were diagnosed from 2009 to 2016 in our Pathology Department. Immunohistochemistry for IDH1 R132H and ATRX, and 1p/19q FISH study were performed in all cases. PCR for IDH1 and 2 was run if needed.

Results: IDH1 R132H mutation was found in $61,5 \%$ of cases (16/26). Nineteen OA grade II (OAII) cases were reclassified into $5 \mathrm{O}$ grade II $1 \mathrm{p} / 19$ codeleted (3 IDH-mutant, 2 IDH-wild type), 6 DA IDH-mutant, 5 DA IDH-wild type, one anaplastic astrocytoma (AA), one Glioblastoma (GBM) IDH-mutant. Only one case was a true OA grade II, with both $1 \mathrm{p} / 19 \mathrm{q}$ codeletion and ATRX mutation. Seven anaplastic OA (OAIII) were reclassified into one anaplastic oligodendroglioma ( $1 \mathrm{p} / 19$ codeleted, IDH-mutant), 2 AA IDH-mutant and 4 GBM (2 IDH-mutant, 2 IDH-wild type). In $23 \%$ of cases of our series $(6 / 26)$ the new diagnosis implied a higher tumor grade, even two cases were upgraded from low to high grade.

Conclusions: According to the new WHO 2016 criteria, in our series $23 \%$ of cases were inaccurately classified and received an inappropriate treatment. It would be suitable for every Pathology Department to retrospectively review recent OA cases, mainly the recently diagnosed cases in which other therapeutic options could be considered.

1749 Prevalence of IgG4-Related Disease in Chronic Orbital Inflammatory Lesions

Juan Rong, Christina Di Loreto, Vivian S Snyder, Jonathan H Lin. University Of California, San Diego, San Diego, CA.

Background: IgG4-related disease is an immune-mediated fibro-inflammatory condition characterized by tumefactive swelling of involved organs, dense lymphoplasmacytic infiltration, markedly increased IgG4+/IgG+ plasma cell ratio, storiform-type fibrosis, and often but not always elevated serum IgG4 concentrations. Orbital structures are among the most common anatomic sites for IgG4-related disease. We studied the prevalence of IgG4-related disease in relation to age, sex and orbital sites in a total of 54 chronic orbital inflammatory lesions identified from our institution.

Design: 54 patients ( 32 female, 22 male, 5 to 92 yo, $87 \%$ over 40 yo) with chronic orbital inflammatory lesions, including 6 lacrimal glands, 18 intraorbital soft tissue/extraocular muscle (EOM) masses, and 30 lacrimal ducts/sacs, were included in our studies. IgG and IgG4 immunostains were performed. IgG+ plasma cells and IgG4+ plasma cells were counted in three high power fields in comparable areas of inflammation and the average IgG4+/IgG+ plasma cell ratio was determined. Lesions with an average IgG4+ IgG+ plasma cell ratio of $30 \%$ or greater were placed in the IgG4-related disease group. The histopathologic features, and when available, serum IgG4 levels, were documented. Results: Orbital lesions from five patients (three male, two female, 54 to 88 yo,) satisfied the criteria of $\mathrm{IgG} 4+/ \mathrm{IgG}+$ plasma cell ratio of $30 \%$ or greater and showed variable degree of fibrosis associated with lymphoplasmacytic infiltration. These include two lacrimal glands, two intraorbital soft tissue/EOM masses, and one lacrimal sac. Four lesions revealed $\mathrm{IgG} 4+/ \mathrm{IgG}+$ plasma cell ratio over $60 \%$. IgG4 serum levels were available in three of the five patients, two with elevated serum IgG4 and one with normal serum IgG4. All three patients responded to steroid therapy. One possible case with $\mathrm{IgG} 4+/ \mathrm{IgG}+$ plasma cell ratio of $20 \%$ was observed in a 46 yo male with an intraorbital soft tissue/EOM mass. Interestingly, markedly increased IgG4+/IgG+ plasma cell ratio was also observed in an intraorbital mass from a 41 yo female with granulomatosis with polyangiitis with positive antineutrophil cytoplasmic antibodies. Conclusions: IgG4-related orbital disease occurred in late middle-aged to elderly patients. More men than women were affected by IgG4-related orbital disease. IgG4related orbital disease was most prevalent in lacrimal glands, followed by intraorbital soft tissue/EOM masses, and least common in lacrimal ducts/sacs. Caution should be practiced in diagnosing IgG4-related orbital disease in patients with granulomatosis with polyangiitis.

1750 Clinicopathological Features of C11orf95-RELA Positive Ependymomas in a Series of Adult and Young Patients with Long FollowUp

Sabrina Rossi, Luisa Toffolatti, Riccardo Signori, Elena Boscato, Lucia Zanatta, Anna G Volpato, Laura Valori, Matilde Cacciatore, Antonella Peciarolo, Stefano Ascani, Cristina Pizzato, Paolo Grotto, Elisabetta Marton, Caterina Giannini, Pierluigi Longatti. Treviso General Hospital, Treviso, Italy; S. Maria Hospital, Terni, Italy; Mayo Clinic, Rochester, MN.

Background: RELA fusion-positive (RELA+) ependymoma, a new WHO 2016 entity, is driven by C11 orf95-RELA fusion leading to the activation of NF-kB pathway and potentially sensitizing the tumor to targeted therapies. RELA+ ependymomas account for $1 / 3$ of all pediatric supratentorial ependymomas (SE) and have a dismal prognosis compared to SE lacking RELA fusion (RELA-). C11 orf95-RELA is associated with clear cell morphology with branching capillaries and L1CAM expression. Little is known regarding RELA+SE occurring in adults. We studied a cohort of 6 adult and 3 young patients ( 1 infant, 2 adolescents), exploring the clinicopathological correlates of C11 orf95-RELA fusion

Design: Formalin fixed paraffin embedded SE were screened for type 1 and 2 C11orf95RELA transcripts by real-time RT PCR, followed by sequencing, and for L1CAM expression by immunohistochemistry. CGH analysis was carried out in 2 RELA+SE with suitable DNA (1 adult, 1 adolescent). A set of 45 non supratentorial ependymomas (nonSE) and 5 subependymomas (subE) were also tested for L1CAM.

Results: C11 orf95-RELA transcript (type 2) was identified in 2/6 adult (33\%) and 2/3 young $(75 \%)$ SE. L1CAM was exclusively expressed in RELA+ SE and was negative in the additional 45 nonSE and 5 subE. Overall, RELA+ and RELA- SE were similar in terms of sex, grade and behavior. In adult SE, clear cells with branching capillaries were seen only in the 2 RELA+SE; instead of the 3 young SE, 1 RELA+ and 1 RELASE displayed this phenotype. Notably, in 1 RELA+ SE, morphology changed over time acquiring an unusually infiltrative growth pattern. Chromotripsis of chr 11 or abnormalities affecting 11q12-q13 region involved in the translocation were found in the 2 RELA+SE analyzed by CGH. Clinicopathological correlations are summarized below

\begin{tabular}{|l|l|l|l|}
\hline & & RELA+ SE & RELA- SE \\
\hline N & & 4 & 5 \\
\hline \multirow{2}{*}{ Age (yrs) } & $\begin{array}{l}\text { Median } \\
\text { (range) }\end{array}$ & $18,5(3-29)$ & $41(17-52)$ \\
\hline Sex & M:F & $2: 2$ & $3: 2$ \\
\hline \multirow{2}{*}{ WHO Grade } & II & 0 & 1 \\
\cline { 2 - 5 } & III & 4 & 4 \\
\hline Clear cells/branching capillaries & Present (\%) & $3(75)$ & $1(20)$ \\
\hline L1CAM & $+(\%)$ & $4(100)$ & $0(0)$ \\
\hline \multirow{2}{*}{ Follow-up time } & $\begin{array}{l}\text { Median } \\
\text { (range) }\end{array}$ & 10 y (5-17) & 7 y (5-12) \\
\hline \multirow{2}{*}{ Recurrence } & YES & $\begin{array}{l}2 \text { (multiple in } 1 \\
\text { case) }\end{array}$ & $\begin{array}{l}3 \text { (multiple in 2 } \\
\text { cases) }\end{array}$ \\
\hline \multirow{2}{*}{ Status at last follow-up } & NED & 3 & 3 \\
\cline { 2 - 5 } & AWD & 1 & 1 \\
\cline { 2 - 5 } & DOD & 0 & 1 \\
\hline
\end{tabular}

Conclusions: In our series, enriched for adult cases and with long follow-up, C11 orf95RELA fusion was not uniformly associated with dismal prognosis. L1CAM may be a surrogate for molecular analysis.

1751 Ki 67 Labeling Index Is Superior to IMP3 in Prediction of Meningioma Recurrence; a Pilot Study

Mohamed M Shareef, Doha E Fayed, Rania E Wasfy, Karima I Marey. Tanta University, School of Medicine, Tanta, Egypt; Kafer El Sheikh General Hospital, Kafer El Sheikh, Egypt.

Background: Recurrence is a major clinical issue of meningiomas. Insulin-like growth factor II mRNA binding protein 3 (IMP3) is a marker of uncontrolled cell proliferation and aggressiveness in many tumors. However, little is known about its relation to meningioma behavior especially recurrence. Ki-67 is a well-known excellent marker for determining the growth fraction and hence biological aggressiveness of tumors. Many authorities advocate this marker for prediction of meningioma recurrence yet with many controversies and different cut-off values. This study aimed to compare the predictive value of IMP3 and $\mathrm{Ki} 67$ in meningioma recurrence.

Design: Forty surgical meningioma specimens were recruited with their clinicopathologic and follow up data. The cases were composed of $28 / 40$ cases $(70 \%)$ of grade I, $9 / 40$ cases $(22.5 \%)$ of grade II and $3 / 40$ cases $(7.5 \%)$ of grade III. The de novo cases were $31 / 40(77.5 \%)$ and the recurrent cases were $9 / 40(22.5 \%)$. The specimens were stained immunohistochemically for IMP3 and Ki 67. Positive IMP3 expression (unequivocal cytoplasmic and/or nuclear staining in more than $5 \%$ of tumor cells) and Ki 67 labeling index (with $4 \%$ cut-off value) were analyzed in relation to the relevant data (age, gender, site, size, recurrence and survival).

Results: Positivity for IMP3 was observed in 32/40 (80\%) of all specimens, in 25/31 $(80.6 \%)$ of de novo specimens and in $7 / 9(77.8 \%)$ of recurrent cases. Significant relations were found between IMP3 and patient gender $(\mathrm{p}=.037)$ and tumor size $(\mathrm{p}=.039)$. IMP3 showed high sensitivity for predicting recurrence $(90 \%)$, low specificity $(26.92 \%)$, Positive Predictive Value (PPV) of $32.14 \%$, Negative Predictive Value (NPV) of $87.5 \%$ and accuracy of 44.44 with overall unacceptable performance as a predictive marker for recurrence in $\mathrm{ROC}$ curve $(\mathrm{AUC}=.588, \mathrm{p}=.417)$. The $\mathrm{Ki}-67$ labeling index was high in $20 / 40(50 \%)$ of all specimens, in $16 / 31(51.6 \%)$ of the de novo specimens and in $4 / 9(44.4 \%)$ of the recurrent specimens. Significant relations were found between $\mathrm{Ki}$ 67 labeling index and tumor grade $(\mathrm{p}=.001)$ and IMP3 expression $(\mathrm{p}=.002)$. Ki 67 labeling index showed sensitivity of prediction of recurrence of $60 \%$, specificity of $53.85 \%$, PPV of $33.33 \%$, NPV of $77.78 \%$ and accuracy of 55.56 with overall acceptable performance $(\mathrm{AUC}=.742, \mathrm{p}=.026)$.

Conclusions: Ki 67 labeling index (with $4 \%$ cut-off value) has a good discriminating power in predicting meningioma recurrence, superior to IMP3 which showed an unacceptable performance as a predictive marker of recurrence. 
1752 Does Molecular Profile Trump Morphology in Glial Neoplasms: An Indian Perspective, Based on the Current WHO Classification (2016) Shivani Sharma, Sambit K Mohanty, Arbind Singh, Anurag Sharma, Beklaswar Salona, Mukesh Kumar, Mohit Kumar, Santosh Pandey, Rahul Katara, Shankar Mohan, Vipin Kumar, Joydeep Mukherjee, Ajay Pandita, Lata Kini. CORE Diagnostics, Gurgaon, India.

Background: Recent advances in genomics, proteomics, and the increasing demands for biomarker validation studies have catalyzed changes in the landscape of neurooncology, as reflected in the most recent molecular classification and nomenclature of central nervous system (CNS) neoplasms. We sought to revisit and apply the current classification in glial neoplasms to evaluate its potential clinical utility.

Design: The study included 151 glial neoplasms (January, 2014 to July, 2016) classified and graded according to WHO Classification, 2007. 1p/19q FISH assay and IDH (IDH1 and IDH2) mutational analysis (polymerase chain reaction-sequencing) were performed. Based on the molecular profile, the tumors were re-classified according to the new WHO classification (2016).

Results: Based on IDH mutation, and 1p/19q co-deletion, and WHO, 2016 criteria, the diagnosis of $24.5 \%$ astrocytic tumors changed to oligodendroglial tumors and $1.3 \%$ oligodendroglial tumors changed to astrocytic tumors. The different tumor categories and their comparison are illustrated in figure 1.

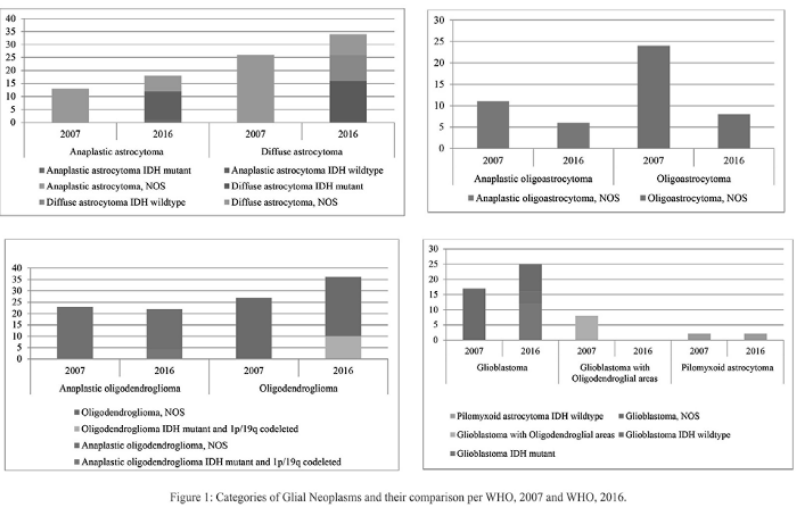

Interestingly, $60 \%$ tumors of oligoastrocytic group could be categorized as pure oligodendroglial or astrocytic. IDH mutation analysis assisted in diagnosing primary (wildtype; $\mathrm{n}=12 ; 48 \%$ ) and secondary (mutant; $\mathrm{n}=4 ; 16 \%$ ) glioblastomas (GBM). Remainder of the GBM cases were of GBM, NOS category. Additionally, eight cases of GBM with oligodendroglial component (GBM-O, WHO, 2007) were re-classified as GBM, NOS $(n=4)$, GBM, IDH mutant $(n=1)$, and GBM, IDH wildtype $(n=3)$.

Conclusions: Our study emphasized the utility of molecular analysis in the classification of the glial neoplasms as pure oligodendroglial or astrocytic in nature, validating and reiterating the new WHO classification.

$60 \%$ tumors of oligoastrocytic group could be categorized as pure oligodendroglial or astrocytic.

GBM and GBM-O categories were further classified as primary and secondary based on their IDH status.

The new classification ramifies further relevant genomic analyses, which have important therapeutic and prognostic implications. Further studies with a larger cohort of patients with clinical follow-up information are warranted to substantiate the current findings.

1753 A Recurrent Kinase Domain Mutation p.D463H in PRKCA Defines Chordoid Glioma of the Third Ventricle

David Solomon, Benjamin Goode, Michael D Hyun, Nancy M Joseph, Jessica Van Ziffle, Nicholas Butowski, Daniel J Brat, Bette Kleinschmidt-DeMasters, Fausto Rodriguez, David N Louis, William Yong, Beatriz Lopes, Marc Rosenblum, Tarik Tihan, Andrew Bollen, Arie Perry. University of California, San Francisco, San Francisco, CA; Emory University, Atlanta, GA; University of Colorado, Denver, CO; Johns Hopkins University, Baltimore, MD; Massachusetts General Hospital, Boston, MA; University of California, Los Angeles, Los Angeles, CA; University of Virginia, Charlottesville, VA; Memorial Sloan Kettering Cancer Center, New York, NY.

Background: Chordoid glioma is a rare brain tumor variant thought to arise from ependymal cells of the lamina terminalis along the anterior wall of the third ventricle. The molecular pathogenesis and optimal treatment strategy for this tumor are unknown. Design: Targeted next-generation sequencing of 480 cancer-associated genes was performed on 10 chordoid gliomas and matched normal tissue. Site-directed mutagenesis was performed on a human PRKCA cDNA. Wild-type or p.D463H mutant PRKCA were expressed in immortalized human astrocytes by lentiviral infection, and cellular phenotypes were assessed including signaling pathway activation and tumorigenic capacity.

Results: A somatic p.D463H missense mutation in PRKCA was identified in all of the ten chordoid gliomas as the solitary and defining genetic alteration. PRKCA, which encodes protein kinase $\mathrm{C}$ alpha, has not been previously identified as a recurrently mutated gene in any human tumor type, although an SLC44A1-PRKCA gene fusion was recently identified in papillary glioneuronal tumors. The p.D463H mutation replaces a critical aspartic acid residue within the active site of the encoded kinase domain that functions as the proton acceptor during ATP hydrolysis. How this recurrent mutation causes chordoid glioma is still under investigation. Our initial functional experiments using lentiviral transduction of wild-type and p.D463H mutant PRKCA into immortalized human astrocytes have not revealed increased phosphorylation of known phosphotargets of protein kinase C (e.g. MARCKS and c-Raf), potentially suggesting a novel gain-of-function caused by this recurrent hotspot mutation.
Conclusions: These studies define a novel genetic biomarker in chordoid glioma of the third ventricle and suggest a promising therapeutic target for this rare brain tumor.

1754 SNP-Based DNA Microarray Analysis May Be More Effective Than Fluorescence In-Situ Hybridization in Identifying BAP1 Alterations in a Uveal Melanoma Cohort

James Solomon, Sarah S Murray, Marie dell'Aquila, John A Thorson, Jonathan H Lin. Univ of California, San Diego, San Diego, CA.

Background: Arising from melanocytes in the choroid, ciliary body, or iris, uveal melanoma (UM) is the most common primary intraocular malignancy in adults. A substantial percentage of UM have an aggressive clinical course and exhibit a propensity to hematogenously metastasize to the liver, while others are indolent and can be treated more conservatively. A few panels of genetic markers exist that can provide prognostic information and stratify tumors based on the chance of metastasis. Monosomy 3 has been classically demonstrated to be an adverse prognostic factor, likely due to loss of one copy of the tumor suppressor gene $B A P 1$. However, other biological mechanisms can inactivate one copy of $B A P 1$, including copy-neutral loss of heterozygosity ( $\mathrm{LOH}$ ). Here, we compare DNA microarray analysis, fluorescence in-situ hybridization (FISH), and next generation sequencing (NGS) in a cohort of UM cases.

Design: From July 2014 to June 2016, enucleation or excision specimens of melanoma involving the orbit from five patients at a single institution were collected. Three of the specimens were clinically diagnosed as primary UM, while two were clinically diagnosed as local invasion of cutaneous melanoma originating from the eyelid. On all five specimens, a genome-wide SNP-based microarray analysis was performed (Oncoscan), as well as a targeted NGS assay of 397 cancer-related genes. In addition, monosomy 3 analysis by FISH was performed on the cases of UM.

Results: In one case of UM, FISH showed the normal two copies of chromosome 3, while Oncoscan identified a copy-neutral LOH at 3p21, the area of the BAPI tumor suppressor gene. For the other two cases of UM, the results were concordant: One case was positive for monosomy 3 and one case was negative for chromosome 3 abnormalities by both modalities. NGS identified GNAQ or GNA11 mutations in all cases of UM and a $B A P 1$ mutation in the case that was positive by both FISH and Oncoscan. The two cases of cutaneous melanoma involving the eye were biologically distinct, with Oncoscan revealing no alterations of 3p21 and NGS showing no GNAQ or GNA11 mutations, but a BRAF V600E mutation in one case.

Conclusions: DNA microarray technology enables a high resolution view of copy number abnormalities and, for SNP-based arrays, includes the ability to identify LOH. Thus, it appears to be more sensitive for detecting alterations that affect the tumor suppressor gene $B A P I$ in UM. In addition, comparing NGS and Oncoscan results of $\mathrm{UM}$ and cutaneous melanoma cases serve to highlight their distinct biology.

1755 MUC4 Is Expressed in Meningioma with High Sensitivity for the Microcystic Variant

Toyohiro Tada, Hisashi Tateyama, Megumi Yoshida, Takashi Matsumoto. Toyokawa City Hospital, Toyokawa, Japan; Kasugai City Hospital, Kasugai, Japan.

Background: MUC4 (Mucin 4, a transmembranous glycoprotein) has recently been identified as a sensitive amd relatively specific marker for sclerosing epithelioid fibrosarcoma/low-grade fibromyxoid sarcoma among mesenchymal tumors. However, anything on MUC4 immunoreactivity in neoplasms of central nervous system is not known yet. We investigated MUC4 expression in meningiomas using immunohistochemistry (IHC).

Design: Formalin-fixed and paraffin-embedded 67 cases of WHO grade I meningioma of central nervous system were retrieved from our pathology files. Hematoxylin-Eosin stained slides of all of them were reviewed to confirm the diagnosis and histological subtype. IHC studies for MUC4, EMA, CD34, STAT-6, and MIB-1 were performed in each subtype. We analyzed and compared IHC findings among the subtypes for MUC4 and EMA.

Results: All the tumors were negative to CD34 and STAT-6. The 67 meningiomas were subtyped ( 26 meningothelial, 6 transitional, 15 fibrous. 15 microcystic, 3 angiomatous and 2 psammomatous). Results of IHC for MUC4 and EMA are summarized in Table 1. Except for fibrous variant, the overall sensitivity of MUC4 and EMA to others were same $(47 / 52,90 \%)$. Although the MUC4-positivity for fibrous variant (33\%) was low, in microcystic variant showed $100 \%$ positivity and the staining score was very high. Five of 67 cases were completely negative for EMA, but instead they were positive for MUC4.

\begin{tabular}{|l|l|l|l|l|l|l|l|}
\hline \multirow{2}{*}{$\begin{array}{c}\text { Subtype of } \\
\text { Meningioma }\end{array}$} & \multirow{2}{*}{$\begin{array}{c}\text { No.of } \\
\text { Cases }\end{array}$} & \multicolumn{2}{|c|}{$\begin{array}{c}\text { IHC: NegativeNo. } \\
(\%)\end{array}$} & \multicolumn{2}{c|}{$\begin{array}{c}\text { IHC: Mildly } \\
\text { PositiveNo. (\%) }\end{array}$} & \multicolumn{2}{c|}{$\begin{array}{c}\text { IHC: Strongly } \\
\text { PositiveNo. (\%) }\end{array}$} \\
\cline { 3 - 9 } & & MUC4 & EMA & MUC4 & EMA & MUC4 & EMA \\
\hline Meningothelial & 26 & $3(12)$ & $2(8)$ & $3(12)$ & $14(54)$ & $20(77)$ & $10(38)$ \\
\hline Transitional & 6 & $0(0)$ & $0(0)$ & $2(33)$ & $1(17)$ & $4(67)$ & $5(83)$ \\
\hline Fibrous & 15 & $10(67)$ & $1(7)$ & $2(13)$ & $6(40)$ & $3(20)$ & $8(53)$ \\
\hline Microcystic & 15 & $0(0)$ & $2(13)$ & $2(13)$ & $9(60)$ & $13(87)$ & $4(27)$ \\
\hline Angiomatous & 3 & $1(33)$ & $0(0)$ & $0(0)$ & $1(33)$ & $2(67)$ & $2(67)$ \\
\hline Psammomatous & 2 & $1(50)$ & $1(50)$ & $1(50)$ & $1(50)$ & $0(0)$ & $0(0)$ \\
\hline Total & 67 & $15(22)$ & $6(9)$ & $10(15)$ & $32(48)$ & $42(63)$ & $29(43)$ \\
\hline
\end{tabular}

Conclusions: MUC4 is preferentially expressed in meningiomas. It is noteworthy that MUC4 is a more sensitive and stronger marker for the microcystic meningioma than EMA. In cases negative for EMA, MUC4 IHC can be alternatively useful for diagnosis of meningioma. 
1756 Is Non-HIV Related DLBCL in the Brain Always Primary? Hammad Tashkandi, Marta E Couce, Mark Cohen. University Hospitals Cleveland Medical Center, Cleveland, $\mathrm{OH}$.

Background: In the absence of HIV infection, diffuse large B-cell lymphoma involving brain parenchyma are believed to represent primary neoplasms without the requirement for systemic staging. Conversely, CNS lesions in patients with extraneural diffuse large B-cell lymphoma are usually ascribed to infectious, autoimmune, and/or therapy related complications.

Design: A search of our pathology archives from 2000-2016 identified 54 parenchymal CNS lymphomas. After clinical and pathological review, patients were divided into two groups: De novo (primary) CNS lymphoma (group 1) and CNS lymphoma preceded by systemic lymphoma (group 2). Additional clinical information obtained included age, gender, race, systemic therapy prior to CNS involvement, HIV status, lymphoma subtype, and time between diagnosis of systemic and CNS lymphoma.

Results: 33 male and 21 female patients ranged in age from 26 to 90 years; 51 of 54 were white. Two cases of CNS and systemic lymphoma were diagnosed synchronously, while seven cases developed metachronously 5 to 9 years following diagnosis of systemic lymphoma. All but 2 cases were negative for HIV the time of diagnosis.

Conclusions: Our retrospective analysis of CNS lymphoma cases showed that intraparenchymal CNS lymphoma arose in the context of systemic lymphoma in 9 of 54 patients $(17 \%)$ with all but 2 of these developing in patients without HIV infection. The possibility of synchronous or metachronous CNS involvement by lymphoma should be considered in the differential diagnosis of these lesions.

1757 Embolic Foreign Material in the Central Nervous System of Children with Heart Disease and a History of Instrumentation: An Update Matthew Torre, Mirna Lechpammer, Audrey Marshall, Amy Juraszek, Robert Padera, Elizabeth Bundock, Sara Vargas, Rebecca Folkerth. Brigham and Women's Hospital, Boston, MA; Children's Hospital Boston, Boston, MA; University of California Davis, Sacramento, CA; Office of the Chief Medical Examiner, Burlington, VT.

Background: Extraordinary interventional procedures are often required to treat pediatric patients with severe, life-threatening cardiopulmonary defects. One underreported complication is embolization of foreign material. We previously reported a series of pediatric postmortem cases demonstrating clinically unsuspected foreign body embolization to the central nervous system (CNS) following invasive diagnostic and therapeutic cardiovascular procedures. In this updated study, we examine the prevalence of embolic foreign material in an expanded cohort and compare this prevalence to a control group. We also investigate a possible source of the embolic material.

Design: Autopsy brain slides for all infants and children with a history of cardiac catheterization, cardiopulmonary bypass, and/or ECMO collected over the course of 5 years at a pediatric tertiary care institution were reviewed for light-microscopic evidence of embolic foreign material. The prevalence of foreign material in this group was compared to a control autopsy group of pediatric patients with no history of cardiovascular procedures. To determine one possible source of embolic foreign material, we reenacted a cutting catheterization procedure on the laboratory bench and evaluated the resultant particulate matter.

Results: Of the 24 patients in the study group (13F, 11M; ages 6 days to 20 years, median 9 months), eight (33.3\%) had foreign material in the CNS in one or more blocks (sampling ranged from 4-19 blocks/case, median 12.5). In contrast, none of the control patients ( $7 \mathrm{~F}, 3 \mathrm{M}$; ages 2 weeks to 19 years, median 8 years) had foreign material in the CNS (sampling ranged from 6-19 blocks/case, median 12.5). In all instances, this material was associated with cellular inflammatory reaction and infarcts. The particulate matter produced during the reenacted catheterization procedure was similar histologically to embolic foreign material identified in a subset of the patients' CNS. Conclusions: Children dying with heart disease and a history of instrumentation have an appreciable risk of foreign material embolization to the CNS. This material can be vaso-occlusive, resulting in infarction. Cardiac catheters are one potential source of this material.

\section{PD-L1 Expression in Sebaceous Carcinoma of the Eyelid} Implicates the Utility of Immune Checkpoint Blockade

John Van Arnam, Jonathan L Curry, Priyadharsini Nagarajan, Phyu P Aung, Alexander $J$ Lazar, Victor G Prieto, Bita Esmaeli, Michael T Tetzlaff. The University of Texas MD Anderson Cancer Center, Houston, TX.

Background: Ocular sebaceous carcinoma (SC) is an aggressive malignancy with high morbidity, frequent metastasis and in a subset of cases, patient death. Surgery remain the mainstay of treatment because effective systemic therapies for locally advanced and metastatic disease are lacking. Immune checkpoint blockade (specifically, inhibition of the endogenous Programmed death protein [PD-1]:Programmed death ligand [PDL1] immunomodulatory signaling axis) has emerged as an effective therapy for many different cancer types, but clinical response depends in part on tumor cell expression of PD-L1. To determine whether SC might be amenable to immune checkpoint blockade, we assessed the expression of PD-L1 and correlated this with the density of associated tumor infiltrating lymphocytes in a series of SCs.

Design: Twelve SCs, including 10 primary ocular sebaceous carcinomas (POSC), 1 paired metastatic ocular sebaceous carcinoma (MOSC) and 1 primary extra-ocular SC (PEOSC) from a total of 11 patients ( 6 men and 5 women) were subjected to immunohistochemical studies with antibodies for PD-L1 (22C3 clone). PD-L1 positivity was scored as \% tumor cells positive. The density of associated tumor infiltrating lymphocytes (TILS) was scored using a sliding scale $(1+, 2+, 3+)$.

Results: Overall, 4/12 (33\%) SCs exhibited positivity for PD-L1. In POSC, 3 of 10 lesions exhibited PD-L1 positivity (range: $3-10 \%$ of tumor cells). One paired MOSC from one patient exhibited a similar degree of positivity as the primary lesion $(3 \%$ of tumor cells). A single PEOSC studied was negative for PD-L1. The tumor associated lymphohistiocytic infiltrate exhibited variable PD-L1 positivity in all cases. No correlation was observed between the density of the TILS and tumor cell expression of PD-L1.

Conclusions: The identification of tumor cell reactivity for PD-L1 in 33\% of SCs implicates immune checkpoint blockade as a potentially effective therapeutic intervention to minimize the morbidity and mortality of a subset of patients with this aggressive ocular malignancy.

1759 Molecular Characterization of a Central Nervous System Inflammatory Myofibroblastic Tumor: Neuropathologists and Molecular Pathologists Adding Value

Jose E Velazquez Vega, Abigail L Goodman, Stewart Neill, Tong Yang, Omid Rouhi, Stephen Hunter, Daniel J Brat, Charles E Hill, Matthew Schniederjan. Emory University Hospital, Atlanta, GA; Children's Hospital of Atlanta, Atlanta, GA.

Background: Inflammatory myofibroblastic tumor (IMT) is a neoplasm of intermediate biologic potential that primarily occurs in the viscera of children and younger adults and exhibits variable morphologic findings. They rarely arise within the central nervous system (CNS). Approximately $50 \%$ of IMTs exhibit ALK receptor tyrosine kinase gene rearrangements. More recently, gene fusions involving other kinases including ROS1, PDGFRB, NTRK3, and RET have been described. The therapeutic implications underscore the importance of thorough molecular characterization of ALK-Negative IMTs

Design: A $25 \mathrm{y} / \mathrm{o}$ male with a 5 year history of a primary CNS IMT presented with a second recurrence causing obstructive hydrocephalus. The tumor initially arose in the posterior fossa, first recurred in the right parietal lobe 19 months after resection and then presented with a second recurrence, an enhancing extra-axial $3 \mathrm{~cm}$ mass associated with the fourth ventricle and superior aqueduct. The patient was chemotherapy and radiotherapy naïve. We performed FISH testing, a cytogenomic microarray and a Sarcoma Fusion Panel (SFP) 26 in the most recent surgical specimen.

Results: All surgical specimens were morphologically identical and comprised of bland myofibroblastic-type spindle cells arranged in a loosely myxoid background with an abundant lymphoplasmacytic infiltrate. Immunohistochemistry for SMA was strongly and diffusely positive within tumor cells. Abundant entrapped islands of brain parenchyma were highlighted with GFAP immunostain. ALK immunohistochemistry was negative and FISH did not detect ALK and RET rearrangements. The SFP 26 identified a TFG-ROS1 gene fusion.

Conclusions: Imperfect correlations between IHC and ALK mutation status in IMTs warrant additional molecular testing to elucidate ALK, ROS1, PDGFRB, NTRK3, and RET alterations that have diagnostic and therapeutic implications given the increasing development of targeted tyrosine kinase inhibitors. The frequency of ROS1 rearrangements in ALK-negative IMTs has not been fully determined yet but is currently estimated to be present in $10 \%$ of IMTs. To date, 13 ROS1 rearranged IMTs have been described and 4 of these had TFG as a fusion partner; none occurred within the CNS. We describe the first ALK-negative CNS IMT with a potentially actionable TFG-ROS1 fusion, providing a possible therapeutic alternative for targeted therapy in this aggressively behaving IMT.

\section{$1760 \quad$ Markers of Progression in Oligodendroglioma}

Jose E Velazquez Vega, Sameer H Halani, Safoora Yousefi, Fatemeh Amrollahi, Chad A Holder, Laila M Poisson, Brent Griffith, Jennifer Eschbacher, Michael Nalisnik, Jeffrey J Olson, Lee AD Cooper, Daniel J Brat. Emory University School of Medicine, Atlanta, GA; Henry Ford Hospital, Detroit, MI; St Joseph's Hospital, Phoenix, AZ Background: Diffuse gliomas are now classified by their molecular genetic alterations, with oligodendrogliomas defined by IDH-mutations and $1 \mathrm{p} / 19 \mathrm{q}$ whole-arm codeletions. Grading schemes derived from the pre-IDH era may not be optimal within the current classification and markers of progression have not been fully defined. Using molecular-genetic and clinical data, along with digitized pathology and MRI features, we investigated markers associated with disease progression in oligodendrogliomas. Design: IDH-mutant, 1p/19q co-deleted gliomas, grades II and III, were selected from The Cancer Genome Atlas (TCGA). Pre-operative MRIs were reviewed for contrast enhancement (CE) and radiographic necrosis (rNEC). Scanned H\&E slides were evaluated for cell density (low-intermediate-high) by histologic review and by a nearest-neighbor algorithm of digitized images. Necrosis (+/-), vascular changes [none-hypertrophy-microvascular proliferation (MVP)], and Ki67 values by IHC were noted. Statistical analysis was performed to identify relationships between features of progression (MRI, histology, Ki-67 indices), genetic alterations and clinical outcomes. Results: Fifty-five tumors had MRIs for review, of which 35 were CE+ and 20 were $\mathrm{CE}-; 10 \mathrm{CE}+$ had rNec. $\mathrm{CE}+$ and $\mathrm{rNec}$ were associated with higher WHO histological grade $(\mathrm{p}<0.001)$, increased Ki-67 expression $(\mathrm{p}=0.003)$, shorter overall survival $(\mathrm{p}=0.04)$, and were enriched for NOTCH1 mutations. Gain of chromosomes $7 \mathrm{p}$ and $11 \mathrm{p}$, and loss of $15 \mathrm{q}$ were copy number alterations (CNAs) exclusively seen in CE+. Among 141 tumors with scanned diagnostic slides, we found that high cell density, defined by histologic review $(\mathrm{p}=0.0835)$ and by computation approaches $(\mathrm{p}=0.0775)$ correlated with a shorter overall survival and were enriched with ZBTB20 mutations $(\mathrm{p}=0.0559)$. NOTCH1 mutations were associated with necrosis $(\mathrm{p}=0.0467)$. Among 89 tumors with Ki67 data, those with with indices $>15 \%$ by IHC were associated with a shorter survival $(\mathrm{p}=0.0153)$ and enriched for PI3K subunit mutations $(\mathrm{p}=0.068)$. Conclusions: Based on our multimodal analysis of molecular, histologic, radiologic and clinical data, we found features of progression in oligodendroglioma, including $\mathrm{CE}+$, high cell density and Ki67 indices and necrosis were associated with specific genetic alterations, including NOTCH1 mutations. Biomarkers of progression will be useful in the stratification of risk for oligodendrogliomas in the molecular era. 
1761 Clinicopathologic Study of Granular Cell Astrocytomas Maria Adelita Vizcaino, Peter C Burger, Fausto Rodriguez. Johns Hopkins University School of Medicine, Baltimore, MD.

Background: Granular cell astrocytoma (GCA) is a rare subtype of infiltrating astrocytic neoplasms usually presenting in adults and associated with a bad prognosis, even in the absence of brisk mitotic activity, microvascular proliferation and/or necrosis. In the current study, we studied our institutional experience with this neoplasm, including clinical, morphologic, immunophenotypic and molecular features, as well as the impact of focal granular cell features in diffusely infiltrating astrocytomas.

Design: A cohort of $23 \mathrm{GCAs}$ was retrospectively evaluated. Clinical information was obtained from the medical records. All the H\&E and immunohistochemical stained slides available were reviewed. $1 \mathrm{p} / 19 \mathrm{q}$ status was evaluated by SNP array in 5 cases. Results: A total of 14 cases of GCA presented in women and 9 in men. Age of presentation ranged from 40 to 81 years (median 59.7 years). Anatomic location was available in 20 cases, with the frontal lobe as the most common site (7/20), followed by the temporal (5/20), parietal (2/20), frontal-parietal (2/20), temporal-parietal (1/20), right hemisphere $(1 / 20)$, splenium $(1 / 20)$ and cerebellum $(1 / 20)$. Granular cell morphology was diffuse in $16(70 \%)$ cases, and focal in $7(30 \%)$ cases. According to WHO criteria for grading of diffuse gliomas, 7 cases were classified as grade II, 8 as grade III, and 8 as grade IV. The neoplastic cells showed variable immunoexpression for GFAP and CD68. MIB-1 proliferation index ranged from low to high. IDH1 (R132H) mutant protein was negative in all the cases tested (7/7), while ATRX expression was consistently retained $(5 / 5)$. Molecular SNP array studies demonstrated partial $1 \mathrm{p}$ deletion with intact $19 \mathrm{q}$ in $3 / 5$ cases, partial $1 \mathrm{p} / 19 \mathrm{q}$ deletions in $1 / 5$ and $19 \mathrm{q}$ whole arm deletion with intact $1 \mathrm{p}$ in $1 / 5$. Whole arm $1 \mathrm{p} / 19 \mathrm{q}$ codeletion typical of oligodendroglial neoplasms was not identified in any case

Conclusions: Granular cell astrocytoma is a unique variant of infiltrating astrocytoma, with WHO grades (II,III,IV) equally distributed. The morphology is usually diffuse but may be focal in a subset. These tumors are characterized by lack of IDH1 (R132H) and preserved ATRX expression. Non-specific 1p19q alterations are frequent and consistent with genomic instability, a finding that deserves further investigation.

\section{Histological Spectrum of Oligodendroglial Tumours: IDH1/2, ATRX and 1p19q}

Amol Wadile, Hetakshi Khurani, Mamta Gurav, Omshree Shetty, Tejpal Gupta, Aliasgar Moiyadi, Prakash Shetty, JayantSastri Goda, Rakesh Jalali, Sridhar Epari. Tata Memorial Centre, Mumbai, Maharashtra, India.

Background: $1 \mathrm{p} 19 \mathrm{q}$ co-deletion is requisite for diagnosis of oligodendroglial tumours. Design: FFPE tissues of histological spectrum of oligodendroglial tumours were evaluated for $1 \mathrm{p} 19 \mathrm{q}$ deletion by FISH, IDHI/2 mutation and ATRX.

Results: 260 oligodendroglial tumours [ODG: 29, ODG-A:129, OA:22, OA-A:68, GBM-O:12] with age range of $19-77$ years. $99.2 \%$ (n: 258) cases were of cerebral hemispheric [53.5\% frontal (n: 138), 14.7\% mixed frontal (n: 38$), 31.8 \%$ non-frontal (n: 82)]. $2(0.08 \%)$ were thalamic. 119 of $158(75.3 \%)$ pure oligodendrogliomas [ODG: 27 and ODG-A: 92], 21 of $90(23.3 \%)$ mixed oligoastrocytic tumours [OA: 6 and OA-A: 15$]$ and 1 of $12(8.3 \%)$ GBM-O were $1 \mathrm{p} 19 \mathrm{q}$ co-deleted. 9 showed isolated $1 \mathrm{p}$ deletion (3.8\%) and 15 showed isolated 19q deletion (5.8\%). $144(65.5 \%)$ pure oligodendrogliomas (ODG: 26 and ODG-A: 118), 71 (32.3\%) oligoastrocytomas (OA: 19 and OA-A: 52$)$ and $5(2.5 \%)$ GBM-O were $I D H I / 2$ mutated [IDHIRI32H:201 (91.4\%); IDHIRI32S: 6 (3.1\%), IDHIRI32C: 2 (1\%), IDHIRI32L:1 (0.5\%) and IDHIR132G: 1(0.5\%), IDH2RI72K: 7 (3.2\%), one each (0.5\%) showed IDH2R172M and $I D H 2 R I 72 \mathrm{~W}$ mutations]. $6.3 \%(9 / 142)$ of pure oligodendroglial cases showed loss of ATRX protein; while $58 \%(45 / 78)$ and $22.2 \%(2 / 22)$ of oligoastrocytic and GBM-O cases showed loss of ATRX protein respectively. The cases were subgrouped into 5 distinct molecular subsets: A (mIDHI/2, 1p19q co-deleted and ATRX retained) - 118 [56.4\%; pure ODG:101(63\%), OAs:17 (18.7\%) and GBM-O:0 ], B (mIDHI/2, 1p19q non-deleted and ATRX loss) - 36 [17.2\%; pure ODG:4(2.5\%), OAs:31(34.1\%) and GBM-O:1); C (mIDH1/2, 1p19q non-deleted and ATRX retained) - 27 [12.9\%; pure ODG:18 (11.2\%), OAs:7 (7.7\%) and GBM-O:2]; D (wIDH1/2,1p19q non-deleted and ATRX retained) - 14 [6.6\%; pure ODG:4, OAs:6 and GBM-O:4]; E (wIDH1/2,1p19q non-deleted and ATRX loss) - 12 [5.7\%; pure ODG:2, OAs:9 and GBM-O:1] Conclusions: Histologically pure oligodendrogliomas can rarely show ATRX protein loss and not all histologically pure oligodendroglial tumours with $\mathrm{mIDHI} / 2$ and retained ATRX protein were $1 \mathrm{p} 19 \mathrm{q}$ co-deleted by FISH. This prudently raises a question of sensitiveness of FISH as technique to detect 1p19q co-deletion or is there any $1 \mathrm{p} 19 \mathrm{q}$ independent alteration existing for oligodendrogliomas.

1763 Molecular and Histologic Features of a Series of Sporadic and Familial Schwannomas

Erik Williams, James Kim, Alona Muzikansky, McKenzie Shaw, John Iafrate, Scott Plotkin, Mario Suva, Anat Stemmer-Rachamimov. Massachusetts General Hospital, Boston, MA; Broad Institute of MIT and Harvard, Boston, MA.

Background: Schwannomas are found either sporadically or as part of a syndrome. Genetic alterations found in schwannomas include those in $N F 2$ and $S M A R C B 1$ (implicated in schwannomatosis), and BRAF V600E. Reduced copy number of chromosome $22 \mathrm{q}$ can be the mechanism for loss of the unaltered allele of $N F 2$ and/or SMARCB1 in some schwannomas.

Design: In this study, we analyzed a cohort of 57 vestibular and non-vestibular schwannomas from three clinical subtypes (Neurofibromatosis 2, schwannomatosis, and sporadic) for reduced copy number ( $N F 2$ and $S M A R C B 1)$, and mutations ( $N F 2$, $S M A R C B 1, N F 1$ and $B R A F)$. Tumors were histologically classified as conventional/ classic, or hybrid. Genomic DNA was extracted from FFPE biopsies. Full coding regions of genes $N F 1, N F 2$, SMARCB1 and BRAF were enriched by anchored multiplex-PCR
(AMP). Resulting libraries were quantitated and sequenced on the Illumina MiSeq System. Bioinformatics analyses were performed using custom scripts. Copy number alteration was detected using a read depth approach after consolidation of single molecule derived reads. INI1 immunohistochemistry was performed. RNA ISH for SMARCB1 was performed.

Results: Five tumors were of hybrid histology, and all five were syndromic. Reduced copy number for $N F 2$ was identified in $33 \%$ of vestibular schwannomas and $18 \%$ of nonvestibular schwannomas. There was no significant difference in reduced copy number of $N F 2$ between sporadic and syndromic forms. BRAF V60OE and NF1 mutations were not identified in any case. For most INI1 mosaics (figure 1), there was no molecular event explaining the partial loss of INI1 IHC.

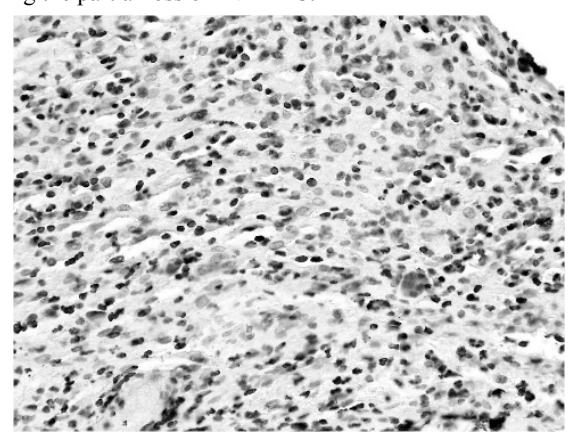

Conclusions: This study confirms the association of hybrid histology with syndromic schwannomas. $N F 1$ mutations did not explain hybrid histology. We did not find $B R A F$ $V 600 E$ mutations in this series. INI1 mosaic pattern was not explained by reduced copy number or mutation of $S M A R C B 1$ as this was found in only a small number of the cases. RNA levels of SMACB1 were evaluated and will be subsequently reported. These results suggest that loss of INI1 expression could be the result of epigenetic alterations.

1764 SSTR2 Is Highly Expressed by Meningiomas: A Novel Diagnostic and Potential Therapeutic Target

Angela Wu, Andrew M Bellizzi. University of Iowa, Iowa City, IA.

Background: Somatostatin, an inhibitory peptide with anti-secretory/proliferative effects, signals through somatostatin receptors (SSTRs). Somatostatin analogues (SAs) octreotide and lanreotide have greatest affinity for SSTR2, making it the most clinically relevant subtype. High-level SSTR2 expression in neuroendocrine (NE) tumors is the basis of somatostatin receptor imaging (SRI), SA therapy, and, very recently, peptide receptor radionuclide therapy (PRRT). Meningiomas were serendipitously found to express SSTRs as early as 1986, and the 1st report of SRI included 2 meningiomas. Current meningioma markers (e.g., EMA, PR) are insensitive, especially in higher-grade lesions, and non-specific. Given recent availability of a reliable monoclonal antibody, we investigated SSTR2 expression in a large cohort of meningiomas and diagnostic mimics. Design: Tissue microarrays were constructed from 177 meningiomas of various types; 19 solitary fibrous tumors/hemangiopericytomas (SFT/HPCs); 10 gliosarcomas; and 91,27 , and 10 consecutive carcinomas, melanomas, and sarcomas metastatic to brain. SSTR2 immunohistochemistry (clone UMB-1) was scored for extent (\%) and intensity $(0-3+)$ and an $\mathrm{H}$-score (extent*intensity) calculated.

Results: SSTR2 was expressed by $95 \%$ of meningiomas, including $97 \% \mathrm{G} 1,95 \% \mathrm{G} 2$, and $86 \%$ G3 tumors. Expression was diffuse, strong (i.e., H-score 200-300), even in higher-grade tumors. SSTR2 was expressed by only $1 / 19$ SFT/HPCs, $1 / 10$ gliosarcomas, $5 / 88$ non-NE carcinomas, and not by any melanomas or sarcomas. SSTR 2 was expressed by $2 / 3 \mathrm{NE}$ carcinomas. Detailed expression data are presented below.

\begin{tabular}{|l|l|l|l|l|}
\hline $\begin{array}{l}\text { Diagnostic } \\
\text { Category }\end{array}$ & Subtype & n & $\begin{array}{l}\text { \% } \\
\text { Positive }\end{array}$ & $\begin{array}{l}\text { Average } \\
\text { H-score (if } \\
\text { positive) }\end{array}$ \\
\hline Meningioma & $\begin{array}{l}\text { NOS (i.e., meningothelial, fibrous, } \\
\text { transitional) }\end{array}$ & 44 & 93 & 216 \\
\hline & Psammomatous & 15 & 100 & 199 \\
\hline & Angiomatous & 10 & 100 & 267 \\
\hline & Microcystic & 8 & 100 & 295 \\
\hline & Secretory & 16 & 100 & 298 \\
\hline & Chordoid & 11 & 91 & 266 \\
\hline & Clear cell & 13 & 100 & 225 \\
\hline & Atypical & 38 & 95 & 208 \\
\hline & Rhabdoid & 6 & 100 & 222 \\
\hline & Anaplastic & 16 & 81 & 226 \\
\hline SFT/HPC & & 19 & 5 & 3 \\
\hline Gliosarcoma & & 10 & 10 & 100 \\
\hline Carcinoma & Non-NE & 88 & 6 & 39 \\
\hline & NE & 3 & 67 & 296 \\
\hline Melanoma & & 27 & 0 & NA \\
\hline Sarcoma & & 10 & 0 & NA \\
\hline
\end{tabular}

Conclusions: SSTR2 is sensitive and specific for meningioma, even among highergrade lesions. Given high-level expression, PRRT should be considered for recurrent tumors having failed standard therapy (i.e., surgery and radiation). 
1765 Study

Hemangioblastoma: A Targeted Next Generation Sequencing

Esther C Yoon, Jian Zhuge, George Kleinman, John T Fallon, Minghao Zhong. New York Medical College at Westchester Medical Center, Valhalla, NY.

Background: Hemangioblastomas (HB) are rare CNS tumors and up to $25 \%$ are associated with von Hippel-Lindau syndrome and biallelic inactivation of VHL genes is an important mechanism for pathogenesis. Same mutation is also seen in clear cell renal cell carcinoma (ccRCC) and part explains the overlap of histomorphological and immunohistochemical characteristics. However, molecular difference between the ccRCC and HB are not yet extensively studied. In this study we looked at common gene mutations by targeted NGS in HB and compared to those in ccRCC.

Design: Our study composed of two sections: 1) analysis of targeted genetic sequence and 2) study of expression of CAIX in hemangioblastoma by IHC and correlate with VHL mutation status. Five cases of hemangioblastom from elderly patients (mean = 53 ) with no known family history of VHL disease were selected. Macrodissection of formalin-fixed paraffin-embedded (FFPE) tissue corresponding to Hematoxylin and Eosin (HE)-stained slides was used to ensure that at least $20 \%$ of the retrieved cells were neoplastic. Genomic DNA was extracted from FFPE tissues by Qiagen AllPrep DNA/RNA Kit. The isolated genomic DNA was subject to targeted sequencing by using Illumina TruSeq Amplicon - Cancer Panel which includes highly cancerassociated 48 genes.

Results: The most common mutation was APC ( $3 / 5$ cases), ATM ( $3 / 5$ cases), ErbB family mutations ( $3 / 5$ cases). The angiogenesis pathway including the genes EGFR and FGFR are seen in $2 / 5$ cases. PTPN11 gene was also found in $2 / 5$ cases. However VHL mutation was not found in any of the cases. However, CAIX was expressed diffuse and strongly in all 5 cases of hemangioblastoma. Therefore, expression of CAIX in absence of VHL mutation raises possibility of alternate interactions of downstream proteins, HIFa and CAIX, EGFR and FGFR.

Conclusions: In this study, we demonstrated multiple genes that are mutated in hemangioblastoma, especially, APC, ATM and ErbB family mutations are highly associated with this tumor. Notably, VHL mutation was not detected among the cases, may indicate VHL structure variant and/or epigenetic changes. Our data demonstrate clearly difference between clear cell renal cell carcinoma and hemangioblastomas at molecular level.

1766 Detection of IDH1/2 and TP53 Mutations and MGMT Methylation in High Grade Gliomas of the Central Nervous System by the Next Generation Sequencing and Pyrosequencing

Yougen Zhan, Tatyana Sidorenko, Aneta Waluszko, David Y Zhang, Fei Ye. Mount Sinai Hospital, New York, NY.

Background: Genetic alternations in gliomas, including IHD1/2 and MGMT promoter methylation, are important markers for treatment plan and prognosis. The next generation sequencing (NGS) offers a convenient means of surveying mutations in common cancer associated genes, including TP53 and IDH1/2 genes. In addition, pyrosequencing provides a sensitive means to detect MGMT promoter methylation.

Design: We retrospectively reviewed twenty-nine patients diagnosed with the CNS gliomas. We subgrouped the patients based on the diagnosis and correlated with the IDH1/2 and P53 mutations as well as MGMT methylation status.

Results: Among 29 patients, 20 were diagnosed with high grade gliomas, including 3 anaplastic oligodendrogliomas (grade III), 8 anaplastic astrocytomas (grade III) and 9 glioblastomas (GBM). Nine patients had low grade gliomas, including 6 astrocytomas (grade I and II) and 3 gangliongliomas (grade I). IDH $1 / 2$ mutations were detected in high grade gliomas, including 4 anaplastic astrocytoma and 3 anaplastic oligodendroglioma (grade III). Only one of 9 GBMs had IDH1 mutation. IDH1/2 mutations detected by the NGS were also compared with IDH immunohistochemistry (IHC) results. IDH mutations were found in all IHC-positive tumors, in contract, IHC was positive in only $50 \%$ tumors with NGS-confirmed IDH mutations. Moreover, MGMT methylation detected in $11(55 \%)$ high grade gliomas. As expected $90 \%$ of TP53 mutations were also found in high grade gliomas.

\begin{tabular}{|l|l|l|l|l|l|}
\hline & $\begin{array}{l}\text { No. of } \\
\text { patients }\end{array}$ & $\begin{array}{l}\text { Average } \\
\text { age in year } \\
\text { (range) }\end{array}$ & $\begin{array}{l}\text { IDH1 } \\
\text { R132H }\end{array}$ & $\begin{array}{l}\text { MGMT } \\
\text { methylation }\end{array}$ & $\begin{array}{l}\text { TP53 } \\
\text { mutation }\end{array}$ \\
\hline Ganglionglioma (Grade I) & 3 & $46(26-73)$ & 0 & 0 & 0 \\
\hline Astrocytoma (Grade I) & 2 & $17(7-27)$ & 0 & 0 & 0 \\
\hline Astrocytoma (Grade II) & 4 & $53(21-84)$ & 0 & 0 & 1 \\
\hline $\begin{array}{l}\text { Anaplastic } \\
\text { oligodendroglioma } \\
\text { (Grade III) }\end{array}$ & 3 & $38.3(34-40)$ & $\begin{array}{l}3^{*} \text { with } \\
\text { an IDH2 } \\
\text { R172K }\end{array}$ & 1 & 0 \\
\hline $\begin{array}{l}\text { Anaplastic astrocytoma } \\
\text { (Grade III) }\end{array}$ & 8 & $36.5(26-67)$ & 4 & 5 & 6 \\
\hline Glioblastoma (Grade IV) & 9 & $53(29-74)$ & 1 & 6 & 3 \\
\hline Total & 29 & & 8 & 12 & 10 \\
\hline
\end{tabular}

Conclusions: Our results showed that NGS is more sensitive and specific to detect IDH1/2 mutations than IHC and should be the method of choice. Moreover, our data demonstrated that IDH1/2 and TP53 mutations and MGMT methylation occur concomitantly in high grade glioma, especially anaplastic oligodendroglioma and astrocytoma, suggesting their interactive roles in the development of high grade glioma.
1767 Prognostic Significance and Characterization of a Small Cell Population in Uveal Melanoma

Pablo Zoroquiain, Evangelina Esposito, Ciro Garcia, Maria A Saornil, William April, Miguel N Burnier. McGill University, Montreal, QC, Canada; Pontificia Universidad Catolica de Chile, Santiago, Chile.

Background: Uveal melanoma is the most frequent primary intraocular tumor. Despite the use of different treatment modalities, $50 \%$ of the patients will develop metastasis, after which the mortality rate is at $90 \%$. Uveal melanoma is composed of two main types of cells: spindle and epithelioid, with the latter associated with a poor prognosis. Nevertheless, there is a subset of small cells located predominately in the infiltrative margins of the tumor and surrounding blood vessels. The aim of the present study is to characterize and evaluate the correlation between the presence of these small cells and clinical outcome.

Design: The clinical and pathological features of 70 patients with uveal melanoma were evaluated. The presence of small cells was quantified as a percentage of the tumor volume (SC). The small cells were characterized using melanocytic markers (HMB-45, Melan A, SOX-10) and stem cell markers (CD133, CD24 and CD38). Univariate and multivariate analysis were conducted to evaluate survival. Clinical details of follow-up visits were available for all patients (mean 148.6 months; SEM 15.1).

Results: For all tumors, SC ranged from 0 to $30 \%$ (median: $1 \%$ ). Thirty-nine tumors $(55.7 \%)$ had SC areas. Univariate analysis showed that the classic cell type $(\mathrm{p}=0.006)$, lymphocytic infiltration $(\mathrm{p}=0.04)$, macrophage infiltration $(\mathrm{p}=0.02)$, ciliary body involvement $(\mathrm{p}=0.02)$ and $\mathrm{SC}$ greater than $5 \%(\mathrm{SC}>5 \%, \mathrm{p}<0.0001)$ had a significant impact on metastasis-free survival. SC was $>5 \%$ in 24 cases; of these, 3 were spindletype and 21 were mixed tumors. Multivariate analysis revealed that a $\mathrm{SC}>5 \%$ was the most significant adverse prognostic factor $(\mathrm{p}<0.001, \mathrm{HR}=4.98)$. Moreover, the small cells were negative for HMB45, focally and weakly positive for MELANA and SOX10 and were negative for stem cell markers.

Conclusions: A high SC is a strong prognostic indicator in patients with uveal melanoma. Based on the loss of melanocytic markers, these cells are less differentiated than the spindle and epithelioid cells and may represent amoeboid transition. Further characterization may lead to new therapeutical targets and a better understanding of the metastatic process in uveal melanoma.

1768 Programmed Cell Death Ligand 1 Expression in Uveal Melanoma Is Associated with Better Patient Outcome and Decreased Tumor Infiltrating Lymphocytes

Pablo Zoroquiain, Evangelina Esposito, Beatriz Dias, Maria A Saornil, Ciro Garcia, Miguel N Burnier. McGill University, Montreal, QC, Canada; Pontificia Universidad Catolica de Chile, Santiago, Chile.

Background: Programmed cell death-1/ligand (PD-1/PD-L1) interaction negatively regulates T-cell activity. PD-L1 expression in tumor cells, antigen-presenting cells and lymphocytes of the tumor microenviroment is associated with response to treatment with PD1/PD-L1 inhibitors, but is still in debate the cutoff value correlated with responders. In uveal melanoma (UM), $40 \%$ of patients will develop liver metastases and $90 \%$ will succumb to their disease. The aim of this study was to analyze PD-L1 as a prognostic marker and as a possible therapeutic target for UM.

Design: Sixty seven enucleated eyes from UM patients with relevant clinical information were analyzed. Immunostaining was performed with the anti-PD-L1 E1L3N clone and with the retinal pigment epithelium as an internal positive control. PD-L1 knockdown in the 92.1 cell line was used as a negative control. Two types of qualifying PD-L1 expression were analyzed: the conventional method that described $>5 \%$ membranous staining in tumor cells, and an alternative that defined positivity as $>5 \%$ in either tumor cell or non-tumor cells (TNT). Cases were evaluated by two ocular pathologists and by using an image analyzer after a 2-hour bleaching protocol. Univariate and multivariate analysis were used to evaluate association of PDL1 with survival, and the kappa score was calculated to compare the agreement between manual and automated evaluation of PD-L1 expression.

Results: PD-L1 expression was positive according to tumor cell and TNT counts in $46.3 \%$ and $55.2 \%$ of the cases, respectively. With univariate analysis, tumor cell and TNT PD-L1 expression were associated with a longer metastasis free survival (MFS) $(P=0.04, \mathrm{P}=0.007)$. However, on multivariate analysis, only TNT positivity was associated with longer MFS $(\mathrm{P}=0.01)$. Furthermore, tumoral and TNT PD-L1 expression was associated with decreased tumor infiltrating lymphocytes (TIL, $\mathrm{P}=0.02$ ). Automated evaluation of TNT showed good agreement with the pathologists' evaluation $(\kappa=0.764)$ and was correlated with longer MFS after both univariate $(\mathrm{P}=0.01)$ and multivariate analysis $(\mathrm{P}=0.02)$

Conclusions: PD-L1 is expressed in uveal melanomas and is associated with better outcome and decreased TIL. These results support the evaluation of anti-PD1/PD-L1 therapy in UM. Automated analysis showed good correlation with conventional methods. Due to its higher objectivity and reproducibility, it is a promising tool that can be utilized in designating a cutoff value for future clinical trials.

\section{Pancreas and Biliary Tree}

\section{Surgical Resection of the Pancreas for Benign Conditions: A} Ten-Year Single Center Retrospective Review

Daniel W Abbott, Kiyoko Oshima. Medilca College of Wisconsin, Milwaukee, WI. Background: Surgical resection of the pancreas (SR) is usually performed when there is clinical suspicion of malignancy. SR is sometimes performed for the management of benign conditions, including chronic pancreatitis, however, cases arise when SR is performed for suspected malignancy and the resulting pathologic diagnosis is benign. The purpose of this study is to investigate SR with benign diagnoses that are suspected to be malignant, with a focus on pancreatitis diagnoses. 